2. To: (Receiving

organization)

SNF CVDE Sub Eroject W441

5. Proj./Prog./Dept./Div.:

W -441

8. Originator Remarks:

Release of FMECA

11. Receiver Remarks: 11A. Design Baseline Document? [] Yes [X] No
3. From: (Originating

organization)

CVDF sub Project W441

6. Design Authority/ Design

Agent/Cog.

Engr.:

Carol C Pitkoff
4. Related EDT No.:

$\mathrm{N} / \mathrm{A}$

7. Purchase Order No.:

$\mathrm{N} / \mathrm{A}$

9. Equip./Component No.:

$\mathrm{N} / \mathrm{A}$

10. System/Bldg./Facility; CVDF

12. Major Assm. Dwg. No.:

$\mathrm{N} / \mathrm{A}$

13. Permit/Permit

Application No.:

$\mathrm{N} / \mathrm{A}$

14. Required Response Date:

$\mathrm{N} / \mathrm{A}$

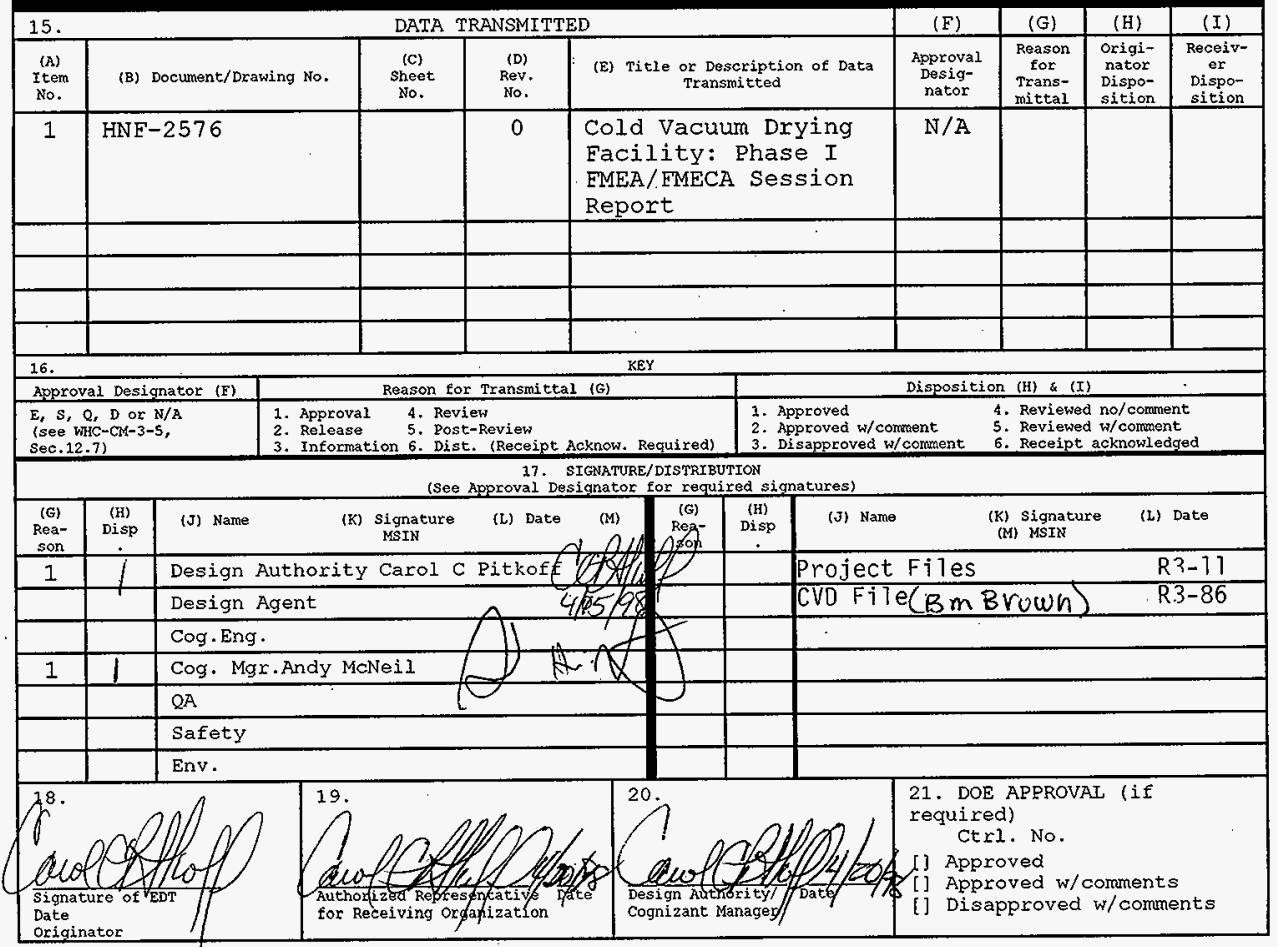

BD-7400-172-2 (05/96) GEE097 


\title{
Cold Vacuum Drying Facility: Phase I FMEA/FMECA Session Report
}

\author{
Carol Pitkoff \\ DE\&S Hanford, Inc., Richland, WA 99352 \\ U.S. Department of Energy Contract DE-AC06-96RL13200
$\mathrm{EDT} / \mathrm{ECN}: 62431 /$
Org Code: $2 \mathrm{~T} 360$
UC: 721
Charge Code: IH302
$B \& R$ Code:
Total Pages: 77

Key Words: Cold Vacuum Drying, FMECA, EMEA

Abstract: Report of the Eailure Modes, Effect, and criticality Analysis performed for the Cold Vacuum Drying process equipment and facility. The report summerized potential problems with the designs, dresign requirments doument, and other baseline documentation.

TRADEMARK DISCLAIMER. Reference herein to any specific commercial product, process, or service by trade name, trademark, manufacturer, or otherwise, does not necessarily constitute or imply its endorsement, recommendation, or favoring by the United states Government or any agency thereof or its contractors or subcontractors.

Printed in the United States of America. To obtain copies of this document, contact: Document Control services, P.0. Box 950, Mailstop H608, Richland WA 99352, Phone (509) 372-2420; Fax (509) 376-4989.
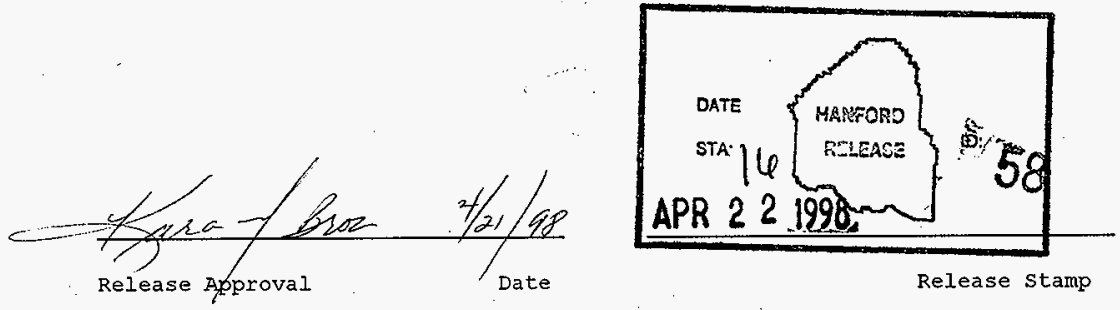

\section{Approved for Public Release}


HNF-2576, Rev. 0

THIS PAGE INTENTIONALLY LEFT BLANK 


\section{SPENT NUCLEAR FUEL \\ COLD VACUUM DRYING FACILITY: \\ PHASE I FMEA/FMECA SESSION}

\section{AUGUST 1997}

Provided for:

Facilitated By:
Carol C. Pitkoff

DE\&S Hanford Inc.

Richland, Washington

Richard A. Harrington, CVS

Lockheed Martin Hanford

Richland, Washington

Geoffrey E. Parker, CVS

ARES Corporation

Richland, Washington 


\section{TABLE OF CONTENTS}

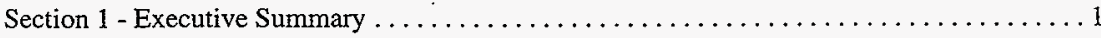

Section 2 - Project Background and Introduction $\ldots \ldots \ldots \ldots \ldots \ldots \ldots \ldots \ldots \ldots \ldots \ldots$

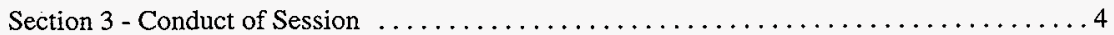

Appendices
A. Scope Statement Sheet
B. Subsystem Final Pass, Actions, Potential Requirement and Design Changes
C. Failure Modes, Effects and Criticality Analysis, Phase I Matrices
D. Session Flipcharts

$H N F-2576$ 


\section{SECTION 1}

\section{EXECUTIVE SUMMARY}

The mission of the Spent Nuclear Fuel (SNF) Project is to remove the fuel currently located in the K-Basins 100 Area to provide safe handling and interim storage of the fuel. The spent nuclear fuel will be repackaged in multi-canister overpacks, partially dried in the Cold Vacuum Drying Facility (CVDF), and then transported to the Canister Storage Building (CSB) for further processing and interim storage.

The CVDF, a subproject to the SNF Project, will be constructed in the 100K area. The CVDF will remove free water and vacuum dry the spent nuclear fuel, making it safer to transport and store at the CSB. At present, the CVDF is approximately $90 \%$ complete with definitive design.

Part of the design process is to conduct Failure Modes, Effects, and Criticality Analysis (FMECA). A four-day FMECA session was conducted August 18 through 21, 1997. The purpose of the session was to analyze 16 subsystems and operating modes to determine consequences of normal, upset, emergency, and faulted conditions with respect to production and worker safety. During this process, acceptable and unacceptable risks, needed design or requirement changes, action items, issues/concerns, and enabling assumptions were identified and recorded. Additionally, a path forward consisting of recommended actions would be developed to resolve any unacceptable risks. The team consisted of project management, engineering, design authority, design agent, safety, operations, and startup personnél from DE\&S Hanford Inc. (DESH), Numatec (NHC), Fluor Daniel Northwest (FDNW), Jason and Associates Co. (JAC), and Merrick Engineering. Technical facilitation was provided by Richard Harrington, CVS, Lockheed Martin Hanford Co. (LMHC), and Jeff Parker, CVS, ARES Corporation. The session successfully met the purpose as stated above.

\section{Session Results}

The session resulted in a Phase I FMECA Matrix, 37 Action Items, nine potential Design Requirement Document changes, and eight potential Design Changes. The most significant issues addressed, within the action items and potential design changes, were identified on the Heating Ventilation and Air Conditioning (HVAC), Process Water Conditioning (PWC), and the Helium (He) subsystems, as follows:

A. HVAC current design and controls for each bay could result in an upset condition that pressurizes the bays. Two action items and one potential design requirement change were identified to resolve this issue. 
B. The PWC system requires a significant redesign due to a factor of 1000 increase in the source term. This redesign, addressed in four action items, will mitigate the increase of potential operator exposure.

C. The He system requires a backup system for uninterrupted supply and in-field verification of He purity and miscellaneous gas constituents in the remaining 5\%. Two action items and one potential design requirement change were identified to resolve this issue.

The process that developed the aforementioned results was an evaluation of 16 subsystems using a Phase I FMECA Matrix. In brief, the matrix was used to identify the likely failure modes (subsystem level), effects of those failures on production and worker safety, and actions required to minimize the likelihood of failure or effects of failures. The key to the worth of the matrix and the success of the session was the objective data known by the team members, or brought to light during the session, and the experience of the team members as they applied the matrix to each subsystem. After the Phase I FMECA Matrix was finished, it was reviewed and analyzed by subsystems to determine if a more detailed Phase II FMECA was needed by component and/or operating mode. As a result, it was recommended that five out of the sixteen subsystems should be subjected to a component level Phase II FMECA at a later date.

The five subsystems requiring a Phase II FMECA were: Vacuum \& Purging System (VPS), PWC, HVAC-B, C, and D. While the entire PWC system was recommended for Phase II, only three operating modes on the VPS and one mode on the three HVAC systems were identified for component level analysis.

The balance of the action items, potential DRDs, and design changes, ranged from upgrading drawings to determining the need for additional instruments and/or components. Recommended design changes resulting from resolution of the action items, potential DRDs, and design changes, will need further evaluation to identify the impacts on capital, operations, and maintenance costs. Once cost is determined, the relative benefit to cost can be determined and a final decision made to make the change or not. That determination will be the subject of separate correspondence.

Due to time constraints the completion dates for actions, potential DRDs, and design changes, will be determined in subsequent meetings and tracked until closure in the on-going project status meetings. 


\section{SECTION 2}

\section{PROJECT BACKGROUND and INTRODUCTION}

The mission of the Spent Nuclear Fuel (SNF) Project is to remove the fuel currently located in the K-Basins 100 Area to provide safe handling and interim storage of the fuel. The spent nuclear fuel will be repackaged in multi-canister overpacks, partially dried in the Cold Vacuum Drying Facility (CVDF), and then transported to the Canister Storage Building (CSB) for further processing and interim storage.

The CVDF, a subproject to the SNF Project, will be constructed in the $100 \mathrm{~K}$ area. Once constructed and turned over to operations, the CVDF will remove free water and vacuum dry the spent nuclear fuel, making it safe to transport and store at the CSB. At present, the CVDF is approximately $90 \%$ complete with definitive design.

Part of the design process is to conduct Failure Modes, Effects, and Criticality Analysis (FMECA). The purpose of a FMECA is to evaluate the system design at the component level to determine possible failure modes and their effects on production and worker safety. Mike Wiemers, Project Manager for the CVDF Project, determined that a facilitated team session would be a good approach for completing a phased approach. This session was limited to a Phase I FMECA on subsystems and some component levels. In addition, the team in this phase would recommend any subsystems and operating modes that would require a Phase II approach, at the component level.

Prior to the session, a blank matrix was developed by the facilitators and several team members for use as a tool prompt and to record information during the session. In addition, 16 subsystems were identified as safety significant and within scope for this Phase I session. 


\section{SECTION 3}

\section{CONDUCT OF SESSION}

Appendix A contains the Scope Statement Sheet and Agenda developed prior to and followed throughout the four day session. The scope statement sheet identifies the team members, and the session scope, objectives, and deliverables. The session began on Monday morning, August 18 , 1997, with a review of the agenda, scope statement sheet, process guidelines and expectations, and opening remarks from the project and deputy project managers. During opening remarks both Mike Wiemers and Carol Pitkoff stressed the importance of building a system that works through the entire life cycle. Specifically, the team was encouraged to remember the system must produce the product needed, at an acceptable rate, and minimizing downtime and any upset conditions. In addition, process emphasis was placed on "recovery thinking" (i.e., what must be done to recover from something that breaks or fails). Following opening remarks the team overviewed the system process and operation.

The Phase I FMECA began with the team reviewing the blank matrix and developing common definitions for each section of the matrix. Following definitions, the team reviewed the list of 16 subsystems and analyzed each subsystem based on failure modes within the subsystem. In some subsystems component failure was considered, but most subsystems were kept at the subsystem level. Typically, the failures were that the system stopped supplying something, supplied too much of something, over pressurized or under pressurized. Once the failure modes were identified they were analyzed using the matrix contained in Appendix. C. During this process any action items, potential Design Requirement Document (DRD) changes, design changes, enabling assumptions, and issues/concerns identified were recorded on corresponding flipcharts. Many of the action items identified will result in DRD or design changes. During this process the team was not certain whether an action was a potential DRD change, design change, or both. In the interest of time it was identified as an action item with impact to be determined later. Thus, a total of 37 actions were recorded. Potential impacts to capital, operation, and/or maintenance costs will be determined as actions are completed.

Once all 16 subsystems were analyzed with the FEMCA matrix, the team reviewed each subsystem matrix for consistency in approach relative to severity class (taking mitigation into account) and to determine if a Phase $\mathrm{M}$ FEMCA would be required. Minor corrections were identified and recorded to ensure that the team's analysis of severity class was consistent. In addition, any identified actions, DRDs, design changes, assumptions, and memory items were recorded. It was noted that a cost trade-off evaluation would be required at a later date based on the subsystem's failure rate, severity class and benefit of a change. Appendix B contains the typed copy of the Subsystem Final Pass/Review, Action Items, Potential DRDs, and Potential Design Changes. 
Finally, a path forward was developed by reviewing and validating the recorded assumptions, issue/concerns, memories, potential DRD changes, potential design changes, and actions. Appendix D contains copies of the reduced Session Flipcharts developed during session and are backup reference to Appendix B and C.:

The session ended with a round robin of each team member answering the questions of last minute items and meeting utility. 


\section{APPENDICES}
A. Scope Statement Sheet And Agenda
B. Subsystem Final Pass/review, Actions Items, Potential Design
Requirements And Design Changes
C. Phase I FMECA Matrix
D. Session Flipcharts 


\section{APPENDIX A}

\section{SCOPE STATEMENT SHEET AND AGENDA}




\section{PROJECT SCOPE STATEMENT SHEET}

Project Title: Cold Vacuum Drying Facility: Phase I FMEA/FMECA Session No. 001 Location of Session: 345 Hills Street, Conference Room 124 Dates: 08/18-21/97

Team Briefing Meeting:

N/A

Date: N/A

\section{$\underline{\text { NAME }}$}

J.J. (John) Irwin (TL)

C.C. (Carol) Pitkoff

R.I. (Dick) Whitehurst

W.C. (Walt) Alaconis

R.A. (Ray) Larson

D.A. (Dan) Reny

C. (Carole) Pili-Vincens

K.J. (Kurt) McCracken

J. (Jim) Robinson

D. (David) Munger

Team Leader (TL)

M.J. (Mike) Wiemers

C.A. (Chris) Thompson

G.P. (Gilles) Chevrier

C.R. (Curtis) Miska
PHONE

376-1639

376-5655

$376-4663$

$376-9390$

373-1698

373-7518

373-1513

373-6653

783-7404

(505) 662-0606
TEAM MEMBERS

MSIN DISCIPLINE CO.

R3-86 CVD Design Authority

R3-86 Project Management

R3-86 I\&C Engineering

R3-85 Operations/Start-up

R3-85 Operations/Start-up

R3-15 Safety Engineering

R3-86 Safety Engineering

S0-04

HVAC Engineering

Design Agent

Design Agent
NHC

DESH

DESH

DESH

DESH

JAC

NHC

FDNW

Merrick

Merrick

\section{FLOATERS}

376-9516

372-0598

373-3609

376-7103
R3-85

R3-85

R3-86

R3-86
Project Manager

Operations/Start-Up

Project Management

HCS Design Authority
DESH

DESH

NHC

NHC

\section{FACILITATORS}

NAME

R.A. (Richard) Harrington

G.E. (Jeff) Parker
PHONE

376-2331

946-3300
MSIN DISCIPLINE

H8-71 Value Engineering

..--...-. Value Engineering
Co.

LMHC ARES

$$
\text { HNF }-2576 \text { Roo } 0
$$




\section{$\underline{\text { SCOPE }}$}

- Cold Vacuum Drying (CVD) Process and Facility Systems: Phase I FMEA/FMECA

- Focus of Phase I is on sub-systems (i.e., CVDF Systems List) and some specific components, as required

- Starting from the point a Multi-Canister Overpack is received with fuel, water, and sludge, and ending, after CVD processing, with the same MCO containing fuel and some water.

\section{OBJECTIVES}

- Overview normal operating process and facility systems sequence

- Describe and list subsystems and/or operating modes to be analyzed.

- Analyze subsystems and/or operating modes to determine consequences of normal, upset, emergency, and faulted conditions with respect to production and worker safety

- Identify acceptable and unacceptable risks, and their basis

- List needed design changes, spare parts, recovery procedures, and estimated recovery time.

- Develop the path forward recommendations and actions to resolve unacceptable risks

\section{DELIVERABLES}

\section{- Draft Phase I FMECA Matrix}

- Listings of needed design changes, spare parts, recovery procedures and estimated recovery times.

- Path Forward Recommendations and Actions 
CVDF Phase I FMEA/FMECA Session

August 18 - 21, 1997

\section{AGENDA}

Day 1

7:30 - Welcome/Introductions/Review Agenda

- Guidelines and Expectations

- Review Scope Statement Sheet (Session Scope, Objectives, and Deliverables)

- Opening Remarks

8:00 - Begin overview and sequencing of normal operating system

- Identify/List subsystems and/or modes on process and facility systems to be analyzed

- Utilize "parking-lot" sheets as required for running information

9:30 - BREAK

9:40 - Conduct Phase I FMECA and matrix on CA/IA and HE sub-systems

11:30 - LUNCH

12:30 - Conduct Phase I FMECA and matrix on FW, and DI sub-systems

2:30 - BREAK

2:40 - Continue FMECA on CHW and VPSCHW sub-systems

4:00 - Finish Day 1 with review of status and Day 2 agenda

\section{Day 2}

7:30 - Review agenda and scope statement sheet

8:00 - Continue FMECA on UPS and Crane/Hoist sub-systems

9:30 - BREAK

9:40 - Continue FMECA on HVACD sub-system

11:30 - LUNCH

12:30 - Continue FMECA on HVACC sub-system

2:30 - BREAK

2:40 - Continue FMECA on HVACB sub-system

4:00 - Finish Day 2 with review of status and Day 3 agenda

$$
\text { HNF-2576 Rowo }
$$




\section{CVDF Phase I FMEA/FMECA Session}

August 18 - 21, 1997

\section{AGENDA}

$\underline{\text { Day } 3}$

7:30 - Review agenda and scope statement sheet

8:00 - Continue FMECA on DCS sub-system

9:30 - BREAK

9:40 - Continue FMECA on PWC sub-system

11:30 - LUNCH

12:30 - Continue FMECA on TWC sub-system

2:30 - BREAK

2:40 - Continue FMECA on TW sub-system

4:00 - Finish Day 3 with review of status and Day 4 agenda

Day 4

7:30 - Review agenda and scope statement sheet

8:00 - Continue FMECA on VPS sub-system

9:30 - BREAK

9:40 - Continue FMECA on EPD sub-system

\section{1:30 - LUNCH}

12:30 - Conduct Subsystem Final Pass/Review

- Identify Phase II Component FMECA Areas

2:30 - BREAK

2:40 - Develop path forward

- Review/Validate running information on parking lot sheets

- Identify actions needed resolve unacceptable risks and proceed forward

4:00 - Finish session with a Round Robin Close-out

- Last minute items

- Meeting utility 


\section{APPENDIX B}

- Subsystem Final Pass/review

- Action Items

- Potential Design Requirement Document Changes

- Potential Design Changes 
Sub-System Final Pass/Review

Sub-System

Actions, DRDs, D.C.s, etc.

Component FMECA

Required?

\begin{tabular}{|c|c|c|}
\hline EPD & See AI\#31 & No \\
\hline VPS & AI \#30, 29/DRD 9/Memories 14 \& 15 & Yes (Modes 1,4,10) \\
\hline TW & AI \#27, 28/Assumption \#7 & No \\
\hline MCS & AI \#21, 22/Assumption \#4,5 \\
\hline PWC & AI \#23, 24, 25, 26/Memories 8, 9/Assumption & Yes (Significant re-design) \\
\hline TWC & 6 & No \\
\hline VPSCHW & None & No \\
\hline UPS & AI\#11 & No \\
\hline CRANES/HOISTS/S.R & None & No \\
\hline HVAC-B & AI \#12/DRD\#6 & Yes (Mode \#2 Control \& Balance) \\
\hline HVAC-C & AI \#18, 19/DRD \#8 & Yes (Mode \#2 Control \& Balance) \\
\hline HVAC-D & DRD \#7/AI \#15, 16 & Yes (Mode \#2 Control \& Balance) \\
\hline CHW & AI\#13, 14, 15, 17/DRD \#7 & No \\
\hline DI & Assumption \#2 & No \\
\hline He & D.C. \#7 & No (Based on AIs, D.C., DRD) \\
\hline CA/A & AI\#8, 9/D.C. \#6/DRD \#5 & No \\
\hline
\end{tabular}

Purpose of Phase 1: Screen systems and subsystems to determine which will be significant contributors to plant down time.

Voting for Phase 2 FMECA:

1. Is system a significant contributor to plant downtime

- Failure rate - frequency of occurrences

- Restoration rate - mean time to restore

2. The "yes" designation indicates that the follow-on reviewers will use the tabulated information to more precisely select the components to do a detailed FMECA. 


\begin{tabular}{|c|c|c|}
\hline \multicolumn{3}{|c|}{ ACTION ITEMS } \\
\hline WHAT & & WHO/WHEN \\
\hline $\begin{array}{l}\text { 1. Determine what the } 25 \text { psig service supplies } \\
\text { (instrument air to HVAC). See Note \#1 }\end{array}$ & $\begin{array}{l}\text { WHO: } \\
\text { WHEN: }\end{array}$ & Merrick - Dave Munger \\
\hline $\begin{array}{l}\text { 2. Finish thermo analysis to verify that shutdown } \\
\text { system results in a safe plant condition and } \\
\text { low corrosion environment (does design } \\
\text { reflect results) }\end{array}$ & $\begin{array}{l}\text { WHO: } \\
\text { WHEN: }\end{array}$ & $\begin{array}{l}\text { Project Team } \\
\text { Ongoing }\end{array}$ \\
\hline $\begin{array}{l}\text { 3. Determine system failure compressor PM rate } \\
\text { vs. demand and plant outage schedule (can } \\
\text { facility last that long [partime] during } \\
\text { compressor maintenance). See Note \#1 }\end{array}$ & $\begin{array}{l}\text { WHO: } \\
\text { WHEN: }\end{array}$ & Merrick - Dave Munger \\
\hline $\begin{array}{l}\text { 4. Develop an operator response to evacuate the } \\
\text { bay on loss of HVAC (TSR consideration) }\end{array}$ & $\begin{array}{l}\text { WHO: } \\
\text { WHEN: }\end{array}$ & Operations - Walt Alaconis \\
\hline $\begin{array}{l}\text { 5. Verify the type of filters in compressed air } \\
\text { dryer. See Note } \# 1\end{array}$ & $\begin{array}{l}\text { WHO: } \\
\text { WHEN: }\end{array}$ & Merrick - Jim Robinson \\
\hline $\begin{array}{l}\text { 6. Verify sight glasses exist for determination of } \\
\text { liquid in the air lines (determine what is in } \\
\text { dryer to help determine bad air). See Note \#1 }\end{array}$ & $\begin{array}{l}\text { WHO: } \\
\text { WHEN: }\end{array}$ & Merrick - Dave Munger \\
\hline $\begin{array}{l}\text { 7. Review potential impact of bad air on safety } \\
\text { class valve operators; review to determine if } \\
\text { an LCO is required (reevaluate severity class } \\
\text { if LCO required) }\end{array}$ & $\begin{array}{l}\text { WHO: } \\
\text { WHEN: }\end{array}$ & $\begin{array}{l}\text { Merrick on review; } \\
\text { Dan Reny/Carol } \\
\text { Pili-Vincens on LCO }\end{array}$ \\
\hline $\begin{array}{l}\text { 8. Determine minimum } \mathrm{He} \text { purity requirement } \\
\text { (Combine actions } 8 \text { and } 9 \text { ). }\end{array}$ & $\begin{array}{l}\text { WHO: } \\
\text { WHEN: }\end{array}$ & John Irwin \\
\hline $\begin{array}{l}\text { 9. Develop method to verify He purity at site } \\
\text { receipt and/or } \mathrm{MCO}\end{array}$ & $\begin{array}{l}\text { WHO: } \\
\text { WHEN: }\end{array}$ & John Irwin \\
\hline $\begin{array}{l}\text { 10. Switch to service water to supply DI water } \\
\text { (design change) }\end{array}$ & $\begin{array}{l}\text { WHO: } \\
\text { WHEN: }\end{array}$ & John Irwin \\
\hline $\begin{array}{l}\text { 11. Determine impact of glycol on MCO and } \\
\text { develop recovery plan, if required; and failure } \\
\text { detection method, if required }\end{array}$ & $\begin{array}{l}\text { WHO: } \\
\text { WHEN: }\end{array}$ & John Irwin/Louie Goldman \\
\hline $\begin{array}{l}\text { 12. Develop recovery procedure }(\mathrm{s}) \text { to } \\
\text { accommodate delays that violate technical } \\
\text { specifications }\end{array}$ & $\begin{array}{l}\text { WHO: } \\
\text { WHEN: }\end{array}$ & Walt Alaconis/George V. \\
\hline $\begin{array}{l}\text { 13. Verify function and need for Damper DMP- } \\
8060 \text {. See Note } \# 1\end{array}$ & $\begin{array}{l}\text { WHO: } \\
\text { WHEN: }\end{array}$ & $\begin{array}{l}\text { Jim Robinson/Kurt } \\
\text { McCracken }\end{array}$ \\
\hline
\end{tabular}




\begin{tabular}{|c|c|}
\hline \multicolumn{2}{|c|}{ ACTION ITEMS } \\
\hline WHAT & WHO/WHEN \\
\hline $\begin{array}{l}\text { 14. Reevaluate the approach to HVAC-D zone } \\
\text { control (HVAC - all, not just D). See Note \#1 }\end{array}$ & $\begin{array}{ll}\text { WHO: } & \text { Jim Robinson/Kurt } \\
& \text { McCracken } \\
\text { WHEN: } & \end{array}$ \\
\hline $\begin{array}{l}\text { 15. Upgrade HVAC drawings to show condensate } \\
\text { drain lines and seal pots }\end{array}$ & $\begin{array}{ll}\text { WHO: } & \text { Jim Robinson/Kurt } \\
& \text { McCracken. } \\
\text { WHEN: } & \\
\end{array}$ \\
\hline $\begin{array}{l}\text { 16. Upgrade HVAC P\&IDs to show: } \\
\text { 1. correct connection of Tank } 4001 \text { to } \\
\text { local exhaust system } \\
\text { 2. correct connection of process skid } \\
\text { vent to local exhaust system } \\
\text { 3. Show the isolation damper in the } \\
\text { process vent system at the bay walls }\end{array}$ & $\begin{array}{l}\text { WHO: Jim Robinson/Kurt } \\
\text { McCracken } \\
\text { WHEN: }\end{array}$ \\
\hline 17. HVAC-D: delete fume hood callouts & $\begin{array}{l}\text { WHO: Merrick - Jim Robinson } \\
\text { WHEN: }\end{array}$ \\
\hline $\begin{array}{l}\text { 18. Verify outside air damper interlock with } \\
\text { general exhaust air flow. See Note \#1 }\end{array}$ & $\begin{array}{l}\text { WHO: Dave Munger/Kurt } \\
\text { McCracken } \\
\text { WHEN: }\end{array}$ \\
\hline $\begin{array}{l}\text { 19. Evaluate the need to provide a "vacuum" } \\
\text { break in the event of loss of air to HVAC-B } \\
\text { (design a change, if required) [safe exit } \\
\text { through door]. See Note \#1 }\end{array}$ & $\begin{array}{l}\text { WHO: Jim Robinson/Kurt } \\
\text { McCracken } \\
\text { WHEN: }\end{array}$ \\
\hline $\begin{array}{l}\text { 20. Evaluate need for local bay repeater alarms } \\
\text { (from problems in other bays). See Note \#1 }\end{array}$ & $\begin{array}{l}\text { WHO: Operations - Walt Alaconis } \\
\text { WHEN: }\end{array}$ \\
\hline 21. Determine needs of programming for MCS & $\begin{array}{l}\text { WHO: Dick Whitehurst } \\
\text { WHEN: }\end{array}$ \\
\hline $\begin{array}{l}\text { 22. Conduct a Design Review on } \\
\text { programs/reprogramming }\end{array}$ & $\begin{array}{l}\text { WHO: Dick Whitehurst } \\
\text { WHEN: }\end{array}$ \\
\hline $\begin{array}{l}\text { 23. Do a dose study on the PWC; determine if } \\
\text { design requires change; generate DRD or } \\
\text { design change as required }\end{array}$ & $\begin{array}{l}\text { WHO: Carol Pitkoff } \\
\text { WHEN: }\end{array}$ \\
\hline $\begin{array}{l}\text { 24. Develop procedures to use installed backup } \\
\text { pumps to recover from PWC loss of pumping } \\
\text { flow (or use pressure control); requires a DRD } \\
\text { change for recognition of requirement. See } \\
\text { Notes } 1 \& 2 \text {. }\end{array}$ & $\begin{array}{l}\text { WHO: Walt Alaconis/John Irwin } \\
\text { WHEN: }\end{array}$ \\
\hline
\end{tabular}




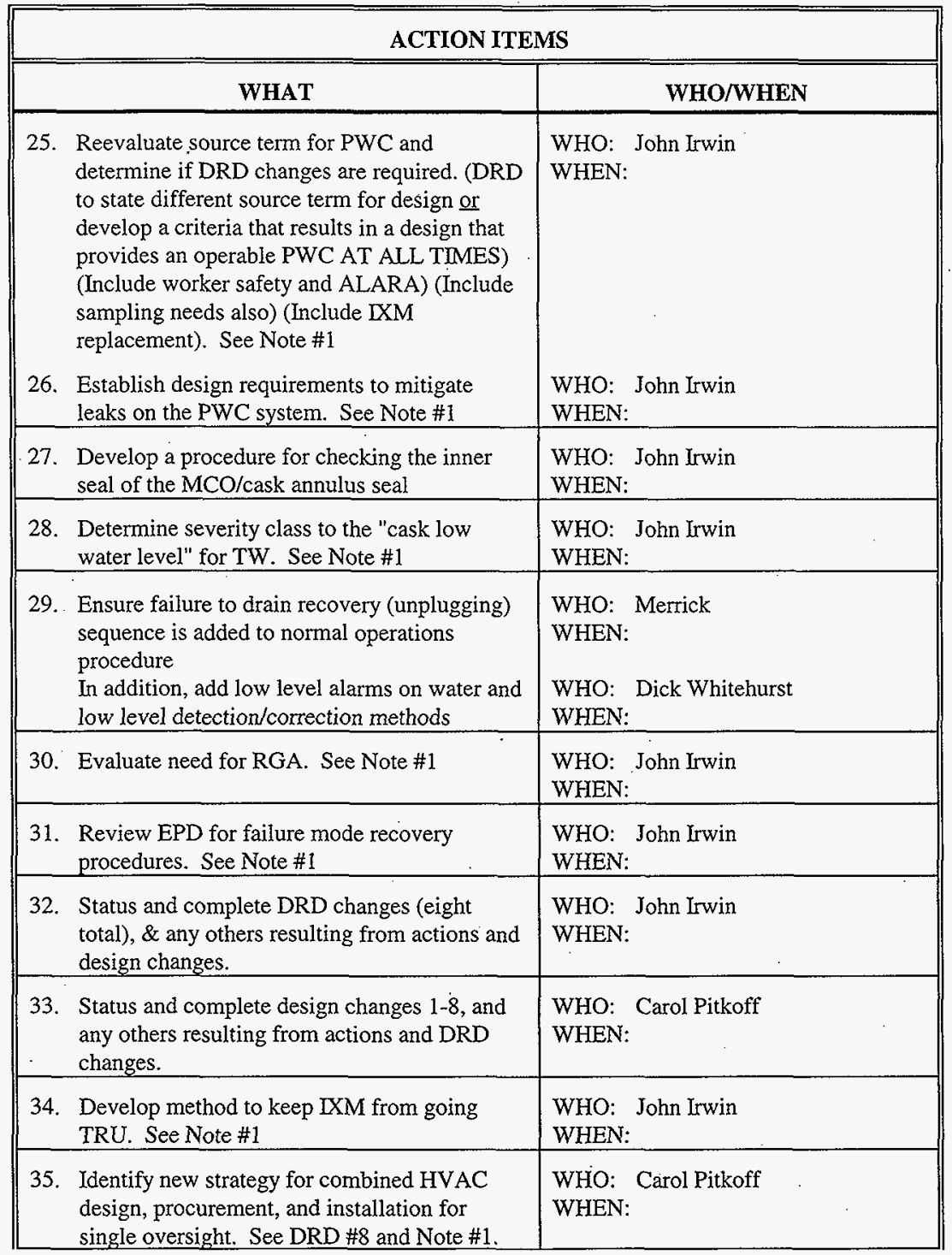




\begin{tabular}{|c|c|}
\hline \multicolumn{2}{|c|}{ ACTION ITEMS } \\
\hline WHAT & WHO/WHEN \\
\hline $\begin{array}{l}\text { 36. Incorporate "plugging of flow meter" in PWC } \\
\text { design. See Note } \# 1\end{array}$ & $\begin{array}{l}\text { WHO: John Irwin/Dave Munger } \\
\text { WHEN: }\end{array}$ \\
\hline $\begin{array}{l}\text { 37. Define normal operating modes for the VPS in } \\
\text { the re-cycle mode, and include in DRD. See } \\
\text { Note \#I }\end{array}$ & $\begin{array}{l}\text { WHO: John Irwin } \\
\text { WHEN: }\end{array}$ \\
\hline
\end{tabular}

\section{Notes:}

1. Using the resulting information, identify any design changes that would minimize the likelihood of failure and/or the effects of the failure. Identify the cost impact and benefit of any recommended design changes. Finally, recommend that change or no change be made. In either case, identify the resulting risk that management is accepting.

2. Consider remote replacement for PWC replacement pump. 


\begin{tabular}{|c|c|c|}
\hline \multicolumn{3}{|c|}{ POTENTIAL DRD CHANGES } \\
\hline WHAT & & WHO/WHEN \\
\hline 1. Project philosophy on spare parts. & $\begin{array}{l}\text { WHO: } \\
\text { WHEN: }\end{array}$ & Walt Alaconis \\
\hline 2. This item was deleted & N/A & \\
\hline $\begin{array}{l}\text { 3. Include capability to put the } \mathrm{MCO} \text { in a cold } \\
\text { shutdown condition (production } \\
\text { consideration--not safety consideration). }\end{array}$ & $\begin{array}{l}\text { WHO: } \\
\text { WHEN: }\end{array}$ & John Irwin \\
\hline $\begin{array}{l}\text { 4. Investigate the dryness criteria on } \\
\text { instrument to determine required dew point } \\
\text { for the cask annulus area. Based on } \\
\text { investigation develop requirement } \\
\text { statement and revise DRD. }\end{array}$ & $\begin{array}{l}\text { WHO: } \\
\text { WHEN: }\end{array}$ & Kurt McCracken \\
\hline $\begin{array}{l}\text { 5. Add: "continuous supply of He" requirement. } \\
\text { - Ensure overpressure or loss of He does not } \\
\text { effect redundant supply trailer. }\end{array}$ & $\begin{array}{l}\text { WHO: } \\
\text { WHEN: }\end{array}$ & John Irwin \\
\hline $\begin{array}{l}\text { 6. Add: Requirements for crane \& rigging } \\
\text { (detect imbalance). }\end{array}$ & $\begin{array}{l}\text { WHO: } \\
\text { WHEN: }\end{array}$ & John Irwin \\
\hline $\begin{array}{l}\text { 7. Establish and add minimum DP } \\
\text { requirements for zone control and alarm in } \\
\text { HVAC systems. }\end{array}$ & $\begin{array}{l}\text { WHO: } \\
\text { WHEN: }\end{array}$ & Kurt McCracken \\
\hline $\begin{array}{l}\text { 8. Potential change - Require a stand alone HVAC } \\
\text { control system with interfaces to MCS (HVAC- } \\
\text { B). See Action Item \#35. }\end{array}$ & $\begin{array}{l}\text { WHO: } \\
\text { WHEN: }\end{array}$ & $\begin{array}{l}\text { Kurt McCracken/ } \\
\text { Dick Whitehurst }\end{array}$ \\
\hline $\begin{array}{l}\text { 9. Identify requirement for } \mathrm{MCO} \text { block values } \\
\text { with respect to particulates in water streams }\end{array}$ & $\begin{array}{l}\text { WHO: } \\
\text { WHËN: }\end{array}$ & John Irwin \\
\hline
\end{tabular}




\begin{tabular}{|c|c|}
\hline \multicolumn{2}{|c|}{ POTENTIAL DESIGN CHANGES } \\
\hline WHAT & WHO/WHEN \\
\hline $\begin{array}{l}\text { Investigate maximum allowable } \\
\text { connection pressure in cask (quick } \\
\text { disconnects attached to cask). See } \\
\text { Note \#1 }\end{array}$ & $\begin{array}{l}\text { WHO: Andy Kee/John Irwin } \\
\text { WHEN: }\end{array}$ \\
\hline $\begin{array}{l}\text { 2. Operating sequence: close } \mathrm{MCO} \\
\text { block valve - when?? (identify } \\
\text { timing) }\end{array}$ & $\begin{array}{l}\text { WHO: Dick Whitehurst/Dan Reny } \\
\text { WHEN: }\end{array}$ \\
\hline $\begin{array}{l}\text { 3. Potential Design Change - Install } \\
\text { check valve in air line to seal ring }\end{array}$ & $\begin{array}{l}\text { WHO: Merrick/John Irwin } \\
\text { WHEN: }\end{array}$ \\
\hline $\begin{array}{l}\text { 4. On Drawing } \mathrm{H}-1-82222 \text {, add } 100 \\
\text { psig service to } \mathrm{rm} 132 ; \text { check if } 25 \\
\text { psig service is needed }\end{array}$ & $\begin{array}{l}\text { WHO: Merrick/John Irwin } \\
\text { WHEN: }\end{array}$ \\
\hline $\begin{array}{l}\text { a. Verify alarm type (PAL 5008) } \\
\text { change as required (need high \& } \\
\text { low pressure). See Note \#1 }\end{array}$ & $\begin{array}{l}\text { WHO: Dick Whitehurst } \\
\text { WHEN: }\end{array}$ \\
\hline $\begin{array}{l}\text { 5. Potential Design Change - Allow } \\
\text { the use of LA for formal purge of } \\
\text { cask annulus. See Note \#1 }\end{array}$ & $\begin{array}{l}\text { WHO: John Irwin } \\
\text { WHEN: }\end{array}$ \\
\hline $\begin{array}{l}\text { 6. Add PT or switch upstream of He } \\
\text { pressure regulator to detect He } \\
\text { supply pressure }\end{array}$ & $\begin{array}{l}\text { WHO: Merrick/Dick Whitehurst } \\
\text { WHEN: }\end{array}$ \\
\hline $\begin{array}{l}\text { 7. Global Change - Review all P\&IDs } \\
\text { for change from PAL to PI }\end{array}$ & $\begin{array}{l}\text { WHO: Merrick/Dick Whitehurst } \\
\text { WHEN: }\end{array}$ \\
\hline 8. 82164 - Change EJR to EDR & $\begin{array}{l}\text { WHO: Merrick/Dick Whitehurst } \\
\text { WHEN: }\end{array}$ \\
\hline
\end{tabular}

\section{Notes:}

1. Using the resulting information, identify any design changes that would minimize the likelihood of failure and/or the effects of the failure. Identify the cost impact and benefit of any recommended design changes. Finally, recommend that change or no change be made. In either case, identify the resulting risk that management is accepting. 


\section{APPENDIX C}

\section{COLD VACUUM DRYING: FAILURE MODES, EFFECTS AND CRITICALITY PHASE I MATRICES}

$$
\begin{gathered}
\text { HNF }-2576 \\
\therefore \operatorname{RoN} 0
\end{gathered}
$$


SNF - Cold Vacuum Drying Project

Date: $8 / 18-21 / 97$

Failure Modes, Effects and Criticality Analysis, Phase I

Sheet No: 1

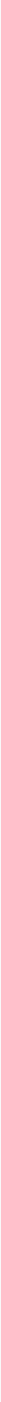


SNF - Cold Vacuum Drying Project

Failure Modes, Effects and Criticality Analysis, Phase I
Date: $8 / 18-21 / 97$

Sheet No: 2

\begin{tabular}{|c|c|c|c|c|c|c|c|c|c|c|c|c|c|}
\hline \multirow{2}{*}{$\begin{array}{l}\text { Subcomponent/ } \\
\text { Subsystem }\end{array}$} & \multirow{2}{*}{$\begin{array}{c}\text { Sub. sys.. } \\
\text { No.J } \\
\text { Component } \\
\text { ID } \\
\end{array}$} & \multirow{2}{*}{$\begin{array}{c}\text { Sub. sys.. } \\
\text { Comp. } \\
\text { Failure } \\
\text { Rate }\end{array}$} & \multirow{2}{*}{$\begin{array}{c}\text { Failure } \\
\text { Mode }\end{array}$} & \multirow{2}{*}{$\begin{array}{l}\text { Alpha } \\
\text { Rank } \\
\text { (TBD) }\end{array}$} & \multicolumn{2}{|c|}{ Failure Effects } & \multirow{2}{*}{$\begin{array}{c}\text { Failure } \\
\text { Detection } \\
\text { Method }\end{array}$} & \multirow{2}{*}{$\begin{array}{l}\text { Mitigation } \\
\text { of Cause }\end{array}$} & \multirow{2}{*}{$\begin{array}{c}\text { Reliability } \\
\text { of } \\
\text { Mitigation }\end{array}$} & \multirow{2}{*}{$\begin{array}{l}\text { Severity } \\
\text { Class }\end{array}$} & \multicolumn{2}{|c|}{ Action(s) Required? } & \multirow[t]{2}{*}{ Remarks } \\
\hline & & & & & Local Effects & End Effects & & & & & Production & $\begin{array}{l}\text { Worker } \\
\text { Safety }\end{array}$ & \\
\hline \multirow[t]{3}{*}{$\mathrm{He}$} & 3.3: & 1 & 105s of Hil & 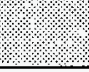 & 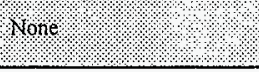 & Loss of prodiction & PT: 5030 & Tone. $1.1 \%$ & N/A & B. & See Renarks & rone: & 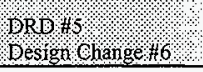 \\
\hline & & EL & Over pressure & & H.P. He \& noise & $\begin{array}{l}\text { Loss of H.E./loss of } \\
\text { production }\end{array}$ & PT. 5030 & $\begin{array}{l}\text { SRV-5027 } \\
\text { SRV-5029 }\end{array}$ & H & B & $\begin{array}{l}\text { See remarks for } \\
\# 1\end{array}$ & None & \\
\hline & & ह1: & Brd & & Tin? & Contaminated gas systen & Mone $1.1 \%$ & 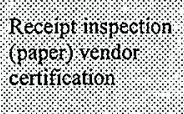 & : 19. & 8 & 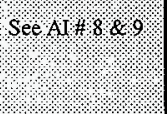 & 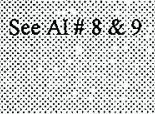 & 4148.89 \\
\hline \multirow[t]{3}{*}{$\mathrm{DI}$} & 25 & $\mathrm{~L}$ & Loss of Demin. & & Potential flooding & potential interruption & PT-9005 & None & N/A & $\mathrm{B}$ & None & $\mathrm{N} / \mathrm{A}$ & Acceptable risk \\
\hline & & 8.18 & 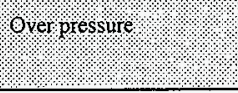 & & spray & rone: & 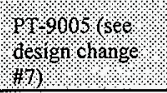 & SRY: $19004: 4$ & i? & s. & None & Wone. & Acceptabilorislly \\
\hline & & $\mathrm{EL}$ & Bad demin. & & None & None & CIT-9007 & Change beds & $\mathrm{H}$ & A & None & None & Acceptable risk \\
\hline \multirow[t]{4}{*}{ CHW } & 473. & ध1: & $\begin{array}{l}\text { loos of coling } \\
\text { capability for process } \\
4\end{array}$ & & Nonel: & 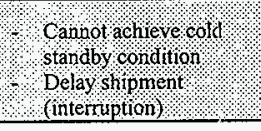 & Tisereral 70\%x & Redingant systern & 1.: & B. & Noner & None? & 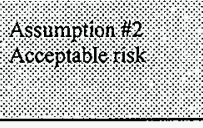 \\
\hline & & EL & Over pressure & & Overflow to drain & None & $\begin{array}{l}\text { PI-7031 \& } \\
7041\end{array}$ & SRV-7050 & $\mathrm{H}$ & A & None & None & Acceptable risk \\
\hline & & 1. & waterleaks & 4 & 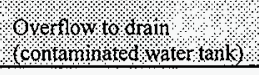 & overfow tank & 489035 & Manivals solitition? & 4. & ॥ & None? & Nono & Aeceptable risk \\
\hline & & EL & $\begin{array}{l}\text { Water leaks } \\
\text { b. Process }\end{array}$ & & None & None & None & None & $\mathrm{N} / \mathrm{A}$ & N/A & None & None & Acceptable risk \\
\hline \multirow[t]{3}{*}{ TWC } & $472 \%$ & 6: & Ioss of cooling process? & & nonie. & $\begin{array}{l}\text { Cannot achieve old } \\
\text { standby \& celay shiprnent }\end{array}$ & $\begin{array}{l}1727053: 8 \\
177054\end{array}$ & Redindantpunps & 1. & 8. & Nones. & roils: & Arceprablel risk? \\
\hline & & EL & Over pressure & & Overflow to floor drain & None & Several PIs & SRV-7063 & $\mathrm{H}$ & A & None & None & Acceptable risk \\
\hline & & 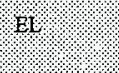 & Whater leaks. process? & & everflown io foror drain & Cannot:achieverold & Severalsis & rone? & itis & 8 & Nonis & wone & Acceptable trist \\
\hline
\end{tabular}


SNF - Cold Vacuum Drying Project

Date: $8 / 18-21 / 97$ Failure Modes, Effects and Criticality Analysis, Phase I

Sheet No: 3

\begin{tabular}{|c|c|c|c|c|c|c|c|c|c|c|c|c|c|}
\hline \multirow{2}{*}{$\begin{array}{l}\text { Subcomponent/ } \\
\text { Subsystem }\end{array}$} & \multirow{2}{*}{$\begin{array}{l}\text { Sub. sys.. } \\
\text { No./ } \\
\text { Component } \\
\text { ID }\end{array}$} & \multirow{2}{*}{$\begin{array}{c}\text { Sub. sys../ } \\
\text { Comp. } \\
\text { Failure } \\
\text { Rate }\end{array}$} & \multirow{2}{*}{$\begin{array}{c}\text { Failure } \\
\text { Mode }\end{array}$} & \multirow{2}{*}{$\begin{array}{l}\text { Alpha } \\
\text { Rank } \\
\text { (TBD) }\end{array}$} & \multicolumn{2}{|c|}{ Failure Effects } & \multirow{2}{*}{$\begin{array}{c}\text { Failure } \\
\text { Detection } \\
\text { Method }\end{array}$} & \multirow{2}{*}{$\begin{array}{c}\text { Mitigation } \\
\text { of Cause }\end{array}$} & \multirow{2}{*}{$\begin{array}{l}\text { Reliability } \\
\text { of } \\
\text { Mitigation }\end{array}$} & \multirow{2}{*}{$\begin{array}{l}\text { Severity } \\
\text { Class }\end{array}$} & \multicolumn{2}{|c|}{ Action(s) Required? } & \multirow[t]{2}{*}{ Remarks } \\
\hline & & & & & Local Effects & End Effects & & & & & Production & $\begin{array}{l}\text { Worker } \\
\text { Safety }\end{array}$ & \\
\hline \multirow[t]{4}{*}{ VPSCHW } & 7.7.4. & 18 & loss of coroling. & & 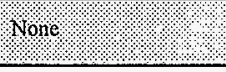 & Increase process tirme? & 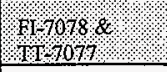 & Redundant punings & 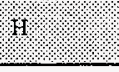 & 8 & Noris: & 4.8n? & Acceptabletisis? \\
\hline & & EL & Over pressure & & Overflow to floor drain & None & $\begin{array}{l}\text { PT-7079 \& } \\
7076\end{array}$ & SRV-7081 & $\mathrm{H}$ & A & None & None & Acceptable risk \\
\hline & 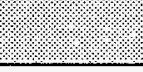 & & Ierks, & & Oyverflowlo floor drair & Loss of cooling & 117078 & Nonie & N/A & 8 & None & ione & Acceptabiler rists \\
\hline & & EL & $\begin{array}{l}\text { Leaks } \\
\text { - Internal }\end{array}$ & & None & $\begin{array}{l}\text { Effluent to VPS \& } \\
\text { potential contamination of } \\
\text { MCO fuel }\end{array}$ & $\begin{array}{l}\text { Potentially } \\
\text { RGA }\end{array}$ & None & $\mathrm{N} / \mathrm{A}$ & $\begin{array}{l}\text { C, } \\
\text { See AI } \\
\# 11\end{array}$ & $\begin{array}{l}\text { Yes } \\
\text { AI \#11 }\end{array}$ & No & See Al \#11 \\
\hline UPS & 93.3 & Et: & 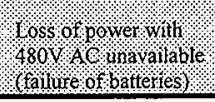 & & rone & $\begin{array}{l}\text { Totalloss (MCS forocess } \\
\text { sops) }\end{array}$ & Plant foes dead & berrodic testing of & 1?. & 18 & Non? & Noner & Acceptable risk \\
\hline \multirow[t]{3}{*}{$\begin{array}{l}\text { Cranes/hoists/seis } \\
\text { mic restraints }\end{array}$} & 14 & $\mathrm{~L}$ & Fail to operate & & none & Delay in process & stops & $\begin{array}{l}\text { periodic } \\
\text { maintenance }\end{array}$ & $\mathrm{H}$ & B & See $A \# 12$ & None & Acceptable risk \\
\hline & & El: & Drop load: & & $\begin{array}{l}\text { Equipinent damage } \\
\text { Personnelinjury }\end{array}$ & Delay in process & Visual ardio & procedures $(08 \%$ & 4. & 8 & $\mathrm{sec}$ & No aditionilial & Acceptablerisls \\
\hline & & $\mathrm{L}$ & $\begin{array}{l}\text { Seismic \#1 failed to } \\
\text { engage/disengage }\end{array}$ & & None & Delay in process & Visual & None & $\mathrm{N} / \mathrm{A}$ & B & See AI \#12 & None & Acceptable risk \\
\hline
\end{tabular}


SNF - Cold Vacuum Drying Project

Failure Modes, Effects and Criticality Analysis, Phase I
Date: $8 / 18-21 / 97$

Sheet No: 4

\begin{tabular}{|c|c|c|c|c|c|c|c|c|c|c|c|c|c|}
\hline \multirow{2}{*}{$\begin{array}{c}\text { Subcomponent/ } \\
\text { Subsystem }\end{array}$} & \multirow{2}{*}{$\begin{array}{c}\text { Sub. sys.. } \\
\text { No.. } \\
\text { Nomponent } \\
\text { ID } \\
\text { ID }\end{array}$} & \multirow{2}{*}{$\begin{array}{c}\text { Sub. sys.. } \\
\text { Comp. } \\
\text { Failure } \\
\text { Rate } \\
\end{array}$} & \multirow{2}{*}{$\begin{array}{l}\text { Failure } \\
\text { Mode }\end{array}$} & \multirow{2}{*}{$\begin{array}{l}\text { Alpha } \\
\text { Rank } \\
\text { (TBD) }\end{array}$} & \multicolumn{2}{|c|}{ Failure Effects } & \multirow{2}{*}{$\begin{array}{c}\text { Failure } \\
\text { Detetion } \\
\text { Method }\end{array}$} & \multirow{2}{*}{$\begin{array}{l}\text { Mitigation } \\
\text { of Cause }\end{array}$} & \multirow{2}{*}{$\begin{array}{l}\text { Reliability } \\
\text { of } \\
\text { Mitigation }\end{array}$} & \multirow{2}{*}{$\begin{array}{l}\text { Severity } \\
\text { Class }\end{array}$} & \multicolumn{2}{|c|}{ Action(s) Required? } & \multirow[t]{2}{*}{ Remarks } \\
\hline & & & & & Local Effects & End Effects & & & & & Production & $\begin{array}{c}\text { Worker } \\
\text { Safety }\end{array}$ & \\
\hline \multirow[t]{3}{*}{ HVAC-D } & 30-4 & EE & 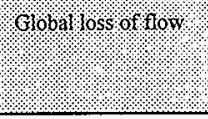 & & Yone & 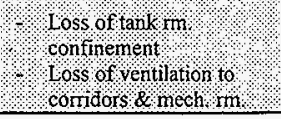 & Aiss-8024 & Redundant fans: & H. & 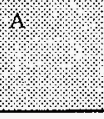 & None & None & 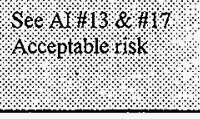 \\
\hline & & M & Loss of zone flow & & None & $\begin{array}{l}\text { Loss of confinement which } \\
\text { may lead to process } \\
\text { shutdown }\end{array}$ & DP \& ref. air & O \& M balancing & M & A & None & $\begin{array}{l}\text { Yes, see Al \#3 } \\
14\end{array}$ & See DRD \#7 \\
\hline & & 1: & Ross of filtration & & None & Tone & Dos test & Tivo fiters instres & is. & is & None: & Nono: & $\begin{array}{l}\text { See-Al ins } \\
\text { Acceptable ist. }\end{array}$ \\
\hline \multirow[t]{4}{*}{ HVAC-B } & $30-2$ & $\mathfrak{L}$ & Loss of flow & & Loss of heating \& cooling & None & PDIS-05 & None & N/A & A & None & None & $\begin{array}{l}\text { Acceptable } \\
\text { See AI } \# \text { 18 } \\
\text { See DRD \#8 }\end{array}$ \\
\hline & & i? & loss of ofutside :ait & & $\begin{array}{l}\text { Brop in pressure } \\
\text { Lncreased DP }\end{array}$ & None & $\mathrm{PPF}: 8 * 20$ & None & N/A & i. & None: & Ses:-1019: & $41+19.19$ \\
\hline & & EL & Over pressurization & & $\begin{array}{l}\text { Excess outside air } \\
\text { Increase in pressure }\end{array}$ & $\begin{array}{l}\text { Loss of confinement DP, } \\
\text { process shutdown }\end{array}$ & PDI- $8 * 20$ & $\begin{array}{l}\text { Fixed manual } \\
\text { controls }\end{array}$ & $\mathrm{H}$ & B & None & None & Acceptable risk \\
\hline & & E: & 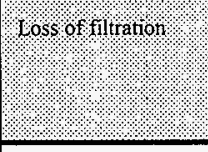 & & None: & None & 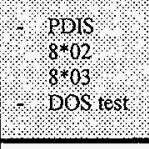 & (itler in sertices & मी. & A & None: & None & Acceptable risk \\
\hline \multirow[t]{3}{*}{ HVAC-C } & $30-3$ & EL & Global loss of flow & & $\begin{array}{l}\text { Loss of flow through } \\
\text { process hood }\end{array}$ & $\begin{array}{l}\text { Potential work area } \\
\text { contamination }\end{array}$ & AMS-8044 & Redundant fans & $\mathrm{H}$ & B & None & None & $\begin{array}{l}\text { See AI \#16 } \\
\text { Acceptable risk }\end{array}$ \\
\hline & & :1: & ioss of zone fion: & & None: & 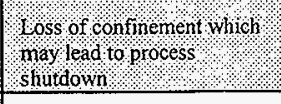 & See prop & mop via propt? & TBo & B. & None: & nonen & SeedRDPH7: \\
\hline & & EL & Loss of filtration & & None & None & DOS test & Two filters in series & $\mathrm{H}$ & A & None & None & $\begin{array}{l}\text { See AI \#15 } \\
\text { Acceptable risk }\end{array}$ \\
\hline
\end{tabular}




\begin{tabular}{|c|c|c|c|c|c|c|c|c|c|c|c|c|c|}
\hline \multirow{2}{*}{$\begin{array}{c}\text { Subcomponent/ } \\
\text { Subsystem }\end{array}$} & \multirow{2}{*}{$\begin{array}{l}\text { Sub. sys.. } \\
\text { No.. } \\
\text { Component } \\
\text { ID }\end{array}$} & \multirow{2}{*}{$\begin{array}{c}\text { Sub. } \\
\text { sys../ } \\
\text { Comp. } \\
\text { Failure } \\
\text { Rate }\end{array}$} & \multirow{2}{*}{$\begin{array}{c}\text { Failure } \\
\text { Mode }\end{array}$} & \multirow{2}{*}{$\begin{array}{l}\text { Alpha } \\
\text { Rank } \\
\text { (TBD) }\end{array}$} & \multicolumn{2}{|c|}{ Failure Effects } & \multirow{2}{*}{$\begin{array}{c}\text { Failure } \\
\text { Detection } \\
\text { Method }\end{array}$} & \multirow{2}{*}{$\begin{array}{c}\text { Mitigation } \\
\text { of Cause }\end{array}$} & \multirow{2}{*}{$\begin{array}{l}\text { Reliability } \\
\text { of } \\
\text { Mitigation }\end{array}$} & \multirow{2}{*}{$\begin{array}{l}\text { Severity } \\
\text { Class }\end{array}$} & \multicolumn{2}{|c|}{ Action(s) Required? } & \multirow[t]{2}{*}{ Remarks } \\
\hline & & & & & Local Effects & End Effects & & & & & Production & $\begin{array}{c}\text { Worker } \\
\text { Safety }\end{array}$ & \\
\hline \multirow[t]{6}{*}{ MCS } & & M & Partial loss of control at i/o & & (sóne: & 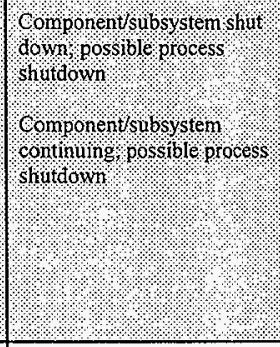 & 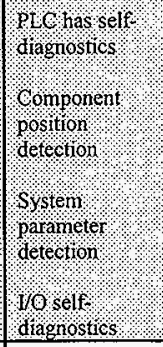 & None: & Nis & $B$ & None: & Nore: & 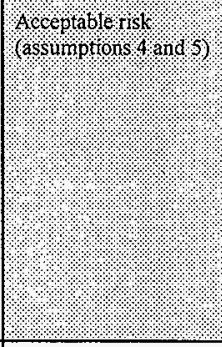 \\
\hline & & L & I/O card fails & & None & Same as above & Same as above & None & N/A & B & None & None & Acceptable risk \\
\hline & & 3 & Pantl fails & & None: & 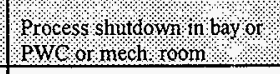 & Sime as above & Nonie: & N/A & B. & None & None: & Acceptable rist: \\
\hline & & EL & $\begin{array}{l}\text { Loss of communications } \\
\text { between the PLC and } \\
\text { computers }\end{array}$ & & \begin{tabular}{|l} 
Alarms \\
Communications errors
\end{tabular} & $\begin{array}{l}\text { Program to go to safe } \\
\text { condition of process after } 60 \\
\text { second time out }\end{array}$ & $\begin{array}{l}\text { Computer } \\
\text { generated alarm } \\
\text { PLC generated } \\
\text { alarm }\end{array}$ & \begin{tabular}{|l}
$\begin{array}{l}\text { Redundant } \\
\text { computers and } \\
\text { cables }\end{array}$
\end{tabular} & $\mathrm{H}$ & B & See AI \#21 & None & Acceptable risk \\
\hline & & $\mathrm{E}$ & Total loss of Mess & & $\begin{array}{l}\text { System goes down every } \\
\text { fhing black }\end{array}$ & $\begin{array}{l}\text { Ail processes are stopped in } \\
\text { safe (failed) corndition? }\end{array}$ & $\begin{array}{l}\text { Visulal and } \\
\text { indible (quit) }\end{array}$ & $\begin{array}{l}\text { Eps and redundant } \\
\text { Systoms: }\end{array}$ & H & B. & None & None & 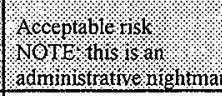 \\
\hline & & M & Bad programming & & $\begin{array}{l}\begin{array}{l}\text { False indication(s) or } \\
\text { control }\end{array} \\
\end{array}$ & Process upset/shutdown & \begin{tabular}{|l} 
Direct and/or \\
indirect alarms
\end{tabular} & $\begin{array}{l}\text { Independent safety } \\
\text { controls; multiple } \\
\text { alarms,; and a } \mathrm{QA} \\
\text { software program }\end{array}$ & $\begin{array}{l}\mathrm{H} \\
\mathrm{H} \\
\mathrm{M}\end{array}$ & B & $\begin{array}{l}\text { Yes, see AI \#'s } \\
22 \text { and } 21\end{array}$ & THD; see AI & \\
\hline
\end{tabular}


SNF - Cold Vacuum Drying Project

Failure Modes, Effects and Criticality Analysis, Phase I
Date: $8 / 18-21 / 97$

Sheet No: 6

\begin{tabular}{|c|c|c|c|c|c|c|c|c|c|c|c|c|c|}
\hline \multirow{2}{*}{$\begin{array}{l}\text { Subcomponent/ } \\
\text { Subsystem }\end{array}$} & \multirow{2}{*}{$\begin{array}{l}\text { Sub. sys.. } \\
\text { No./ } \\
\text { Component } \\
\text { ID }\end{array}$} & \multirow{2}{*}{$\begin{array}{l}\text { Sub. sys..' } \\
\text { Comp. } \\
\text { Failure } \\
\text { Rate }\end{array}$} & \multirow{2}{*}{$\begin{array}{c}\text { Failure } \\
\text { Mode }\end{array}$} & \multirow{2}{*}{$\begin{array}{l}\text { Alpha } \\
\text { Rank } \\
\text { (TBD) }\end{array}$} & \multicolumn{2}{|c|}{ Failure Effects } & \multirow{2}{*}{$\begin{array}{c}\text { Failure } \\
\text { Detection } \\
\text { Method }\end{array}$} & \multirow{2}{*}{$\begin{array}{l}\text { Mitigation } \\
\text { of Cause }\end{array}$} & \multirow{2}{*}{$\begin{array}{c}\text { Reliability } \\
\text { of } \\
\text { Mitigation }\end{array}$} & \multirow{2}{*}{$\begin{array}{l}\text { Severity } \\
\text { Class }\end{array}$} & \multicolumn{2}{|c|}{ Action(s) Required? } & \multirow[t]{2}{*}{ Remarks } \\
\hline & & & & & Local Effects & End Effects & & & & & Production & $\begin{array}{l}\text { Worker } \\
\text { Safety }\end{array}$ & \\
\hline \multirow[t]{6}{*}{ PWC } & : $14:$ & & loss & & Increased adiation & 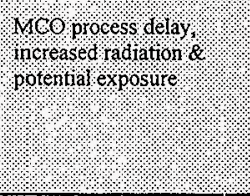 & 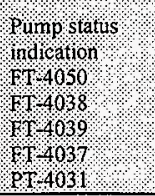 & Wone: : : & $\sqrt{1 / 19}$ : & $\sqrt{1}+4$ & 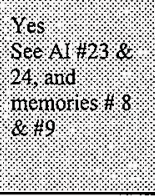 & Ses Ar" & \\
\hline & & $\mathrm{H}$ & Loss of IXM capability & & Increased radiation & $\begin{array}{l}\text { MCO process delay, } \\
\text { increased radiation \& } \\
\text { potential exposure }\end{array}$ & $\begin{array}{l}\text { PDISH- } 4044 \\
\text { or } 4041 \\
\text { Sampler results } \\
\text { FT-4039 } \\
\end{array}$ & Sampling & $\mathrm{EL}$ & B & $\begin{array}{l}\text { Yes } \\
\text { See AI \#25 }\end{array}$ & $\begin{array}{l}\text { Yes } \\
\text { See AI \#25 }\end{array}$ & Assumption \#6 \\
\hline & & y. & 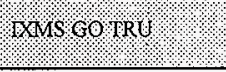 & & nonie & Delayprocess & Salnipling & $\begin{array}{l}\text { Sampling : } 8 \text {. } \\
\text { analysis foequency. }\end{array}$ & E. & B & 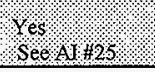 & Y.s. & Assumptionit6 6 \\
\hline & & $\mathrm{L}$ & $\begin{array}{l}\text { Loss of transfer } \\
\text { capability }\end{array}$ & & $\begin{array}{l}\text { Potential for increased } \\
\text { radiation }\end{array}$ & $\begin{array}{l}\text { Production delays due to } \\
\text { high/full tank }\end{array}$ & $\begin{array}{l}\text { LI-4016 } \\
\text { LI-4014 }\end{array}$ & None & NA & B & $\begin{array}{l}\text { Yes } \\
\text { See AI \#24 }\end{array}$ & None & $\begin{array}{l}\text { Accept the risk of no } \\
\text { redundant pump for P- } 00\end{array}$ \\
\hline & & il & ? & & 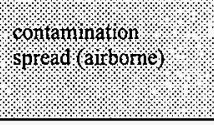 & Belay process & 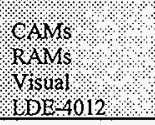 & Propd nantenance & 1. & ?. & Yres.ar: & Yes Au & \\
\hline & & EL & Spray & & $\begin{array}{l}\text { contamination } \\
\text { spread (airborne) }\end{array}$ & $\begin{array}{l}\text { Delay process } \\
\text { Potential exposure }\end{array}$ & $\begin{array}{l}\text { CAMs } \\
\text { RAMs } \\
\text { Visual } \\
\text { LDE-4012 }\end{array}$ & Proper maintenance & $\mathrm{H}$ & $\mathrm{C}$ & $\begin{array}{l}\text { Yes } \\
\text { See AI \#26 }\end{array}$ & $\begin{array}{l}\text { Yes } \\
\text { See AI } \# 26\end{array}$ & \\
\hline
\end{tabular}


SNF - Cold Vacuum Drying Project

Date: 8/18-21/97 Failure Modes, Effects and Criticality Analysis, Phase I

Sheet No: 7

\begin{tabular}{|c|c|c|c|c|c|c|c|c|c|c|c|c|c|}
\hline \multirow{2}{*}{$\begin{array}{l}\text { Subcomponent/ } \\
\text { Subsystem }\end{array}$} & \multirow{2}{*}{$\begin{array}{l}\text { Sub. sys.. } \\
\text { No./ } \\
\text { Component } \\
\text { ID }\end{array}$} & \multirow{2}{*}{$\begin{array}{l}\text { Sub. } \\
\text { sys... } \\
\text { Comp. } \\
\text { Failure } \\
\text { Rate } \\
\end{array}$} & \multirow{2}{*}{$\begin{array}{l}\text { Failure } \\
\text { Mode }\end{array}$} & \multirow{2}{*}{$\begin{array}{l}\text { Alpha } \\
\text { Rank } \\
\text { (TBD) }\end{array}$} & \multicolumn{2}{|c|}{ Failure Effects } & \multirow{2}{*}{$\begin{array}{c}\text { Failure } \\
\text { Detection } \\
\text { Method }\end{array}$} & \multirow{2}{*}{$\begin{array}{l}\text { Mitigation } \\
\text { of Cause }\end{array}$} & \multirow{2}{*}{$\begin{array}{l}\text { Reliability } \\
\text { of } \\
\text { Mitigation }\end{array}$} & \multirow{2}{*}{$\begin{array}{l}\text { Severity } \\
\text { Class }\end{array}$} & \multicolumn{2}{|c|}{ Action(s) Required? } & \multirow[t]{2}{*}{ Remarks } \\
\hline & & & & & Local Effects & End Effects & & & & & Production & $\begin{array}{l}\text { Worker } \\
\text { Safety }\end{array}$ & \\
\hline \multirow[t]{6}{*}{ TW } & $47+$ & 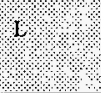 & T.oss of llon' & & Nons & Processing delay and will & PT $3+108$ & rone: & N/\% & 8 & None & Nonere & 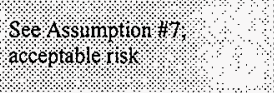 \\
\hline & & EL & 2. Over pressure & & $\begin{array}{l}\text { Loss of seal; system drains } \\
\text { through seal }\end{array}$ & $\begin{array}{l}\text { System shutdown/failure; } \\
\text { will not achieve cold } \\
\text { standby }\end{array}$ & $\begin{array}{l}\text { PT-3*08 } \\
\text { FE-3*16 }\end{array}$ & $\begin{array}{l}\text { FCV- }-3 * 8 \text { and } \\
\text { various interlocks }\end{array}$ & $\mathrm{H}$ & B & None & None & Acceptable risk \\
\hline & & 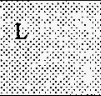 & 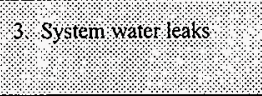 & & Potential contamination & Delay in process & Yisualland & Posting before each & E: & B. & Yे: & 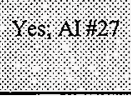 & \\
\hline & & $\mathrm{EL}$ & 4. Cask low water level & & None & Process shutdown & *S.C. indication & $\begin{array}{l}\text { S.C. design } \\
\text { prevention }\end{array}$ & $\mathrm{H}$ & ${ }^{*} \mathrm{C}$ & None & None & *See AI \#28 \\
\hline & & 1,18 & 5 Bad water/: & & Potentilaladiation & None? & None: & None: & \%/, & A. & None: & None: & 4 cceptable risls \\
\hline & & EL & $\begin{array}{l}\text { 6. Loss of temperature } \\
\text { control (high/low) }\end{array}$ & & None & Process delays & $\begin{array}{l}\text { TT-3*05 } \\
\text { S.C. TSH }\end{array}$ & $\begin{array}{l}\text { S.C. prevention and } \\
\text { normal prevention }\end{array}$ & $\mathrm{H}$ & B & None & None & Acceptable risk \\
\hline \multirow[t]{4}{*}{ VPS } & 07. & $\begin{array}{l}\text { Valves } \\
\text { plugging }\end{array}$ & 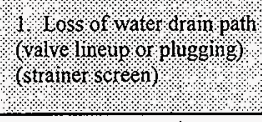 & & Increased radiation & 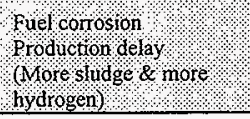 & Itir & $\begin{array}{l}\text { Valves-rione } \\
\text { liugging-correct } \\
\text { loading }\end{array}$ & $\sqrt{14}$ & : & Seest: 1 is? & & \\
\hline & & $\mathrm{M}$ & $\begin{array}{l}\text { 1a. Excessive returned } \\
\text { water }\end{array}$ & & None & $\begin{array}{l}\text { Process delay (long, long } \\
\text { drying time) }\end{array}$ & See AI \#29 & $\begin{array}{l}\text { Fully plugged-none } \\
\text { Slow drain-multiple } \\
\text { drain cycles }\end{array}$ & $\begin{array}{l}\mathrm{N} / \mathrm{A} \\
\mathrm{EL}\end{array}$ & A & $\begin{array}{l}\text { Yes; see } \\
\text { AI } \# 29\end{array}$ & None & \\
\hline & & & 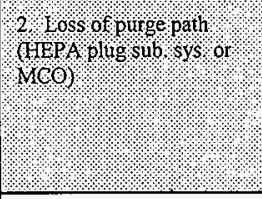 & & & 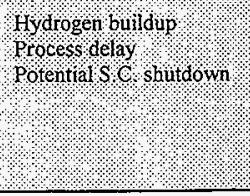 & 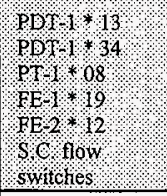 & None & N\% & B: & 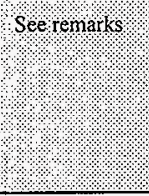 & Seerlemarls: & 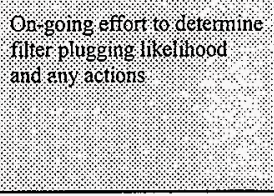 \\
\hline & & $\mathrm{EL}$ & $\begin{array}{l}\text { 3. Loss of DI rinse } \\
\text { (valves) }\end{array}$ & & Increased radiation & $\begin{array}{l}\text { Process delay } \\
\text { MCO plug valve seal } \\
\text { damaged or not reseating }\end{array}$ & $\begin{array}{l}\text { Valve position } \\
\text { alarms }\end{array}$ & None & $\mathrm{N} / \mathrm{A}$ & B & $\begin{array}{l}\text { Yes; see DRD } \\
\text { \#9 (and issue) }\end{array}$ & None & \\
\hline
\end{tabular}


SNF - Cold Vacuum Drying Project

Date: $8 / 18-21 / 97$ Failure Modes, Effects and Criticality Analysis, Phase I

Sheet No: 8

\begin{tabular}{|c|c|c|c|c|c|c|c|c|c|c|c|c|c|}
\hline \multirow{2}{*}{$\begin{array}{l}\text { Subcomponent/ } \\
\text { Subsystem }\end{array}$} & \multirow{2}{*}{$\begin{array}{c}\text { Sub. sys.. } \\
\text { No.I } \\
\text { Component } \\
\text { ID }\end{array}$} & \multirow{2}{*}{$\begin{array}{l}\text { Sub. } \\
\text { sys.. } \\
\text { Comp. } \\
\text { Failure } \\
\text { Rate } \\
\end{array}$} & \multirow{2}{*}{$\begin{array}{l}\text { Failure } \\
\text { Mode }\end{array}$} & \multirow{2}{*}{$\begin{array}{l}\text { Alpha } \\
\text { Rank } \\
\text { (TBD) }\end{array}$} & \multicolumn{2}{|c|}{ Failure Effects } & \multirow{2}{*}{$\begin{array}{c}\text { Failure } \\
\text { Detection } \\
\text { Method }\end{array}$} & \multirow{2}{*}{$\begin{array}{l}\text { Mitigation } \\
\text { of Cause }\end{array}$} & \multirow{2}{*}{$\begin{array}{l}\text { Reliability } \\
\text { of } \\
\text { Mitigation }\end{array}$} & \multirow{2}{*}{$\begin{array}{l}\text { Severity } \\
\text { Class }\end{array}$} & \multicolumn{2}{|c|}{ Action(s) Required? } & \multirow[t]{2}{*}{ Remarks } \\
\hline & & & & & Local Effects & End Effects & & & & & Production & $\begin{array}{c}\text { Worker } \\
\text { Safety }\end{array}$ & \\
\hline \multirow[t]{7}{*}{ VPS } & 07 & 4. & $\begin{array}{l}\text { 4. Lossor vaccirim } \\
\text { (pimpsand path) }\end{array}$ & & roro: & process delay & $\begin{array}{l}\text { P1:108, } 110 \\
208, \text { and } 209\end{array}$ & None: & $\mathrm{N} / \mathrm{s}$ & 8. & See memory & None & \\
\hline & & EL & 5. Loss of condenser & & None & Process delay & $\begin{array}{l}\mathrm{TT}-2 * 35 \& \\
36\end{array}$ & None & $\mathrm{N} / \mathrm{A}$ & $\mathrm{A}$ & None & None & Acceptable risk \\
\hline & & $\sqrt{2}: 3$ & 6. Loss of cicrailin path. & & Rootedtial over flow & 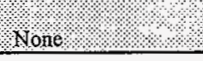 & $1 \mathrm{ssH} 2,25$ & None & N: & 2. & Nonte- & None: & Acceplabil. isk 1 , 1 \\
\hline & & $\ldots$ & 7. Loss of blower and path & & None & None & & & & & & & See memory item $\# 15$ \\
\hline & & t. & 8. Loss of RGA & & None? & 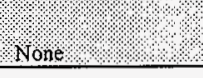 & $\mathrm{AE} 22 \% 13$ & Nones & $\mathrm{N} / \mathrm{A}$ & A & None & Nonice. & 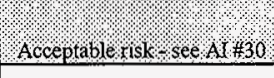 \\
\hline & & EL & 9. Loss of filtration & & Increased radiation & Process delay & None & None & $N / A$ & $\mathrm{~B}$ & None & None & Acceptable risk \\
\hline & & $\sqrt{4}$ & 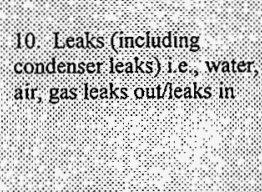 & & Potential contantination & process stiutdown & 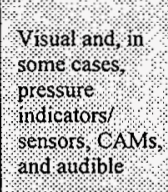 & $\begin{array}{l}\text { rone } \\
\text { (other than initial } \\
\text { startup tests) }\end{array}$ & Nis. & 8. & rone & rone & Acceptablerist: \\
\hline
\end{tabular}




\section{APPENDIX D}

SESSION FLIPCHARTS 


\section{FACLUTATED TEAM SESSLON}

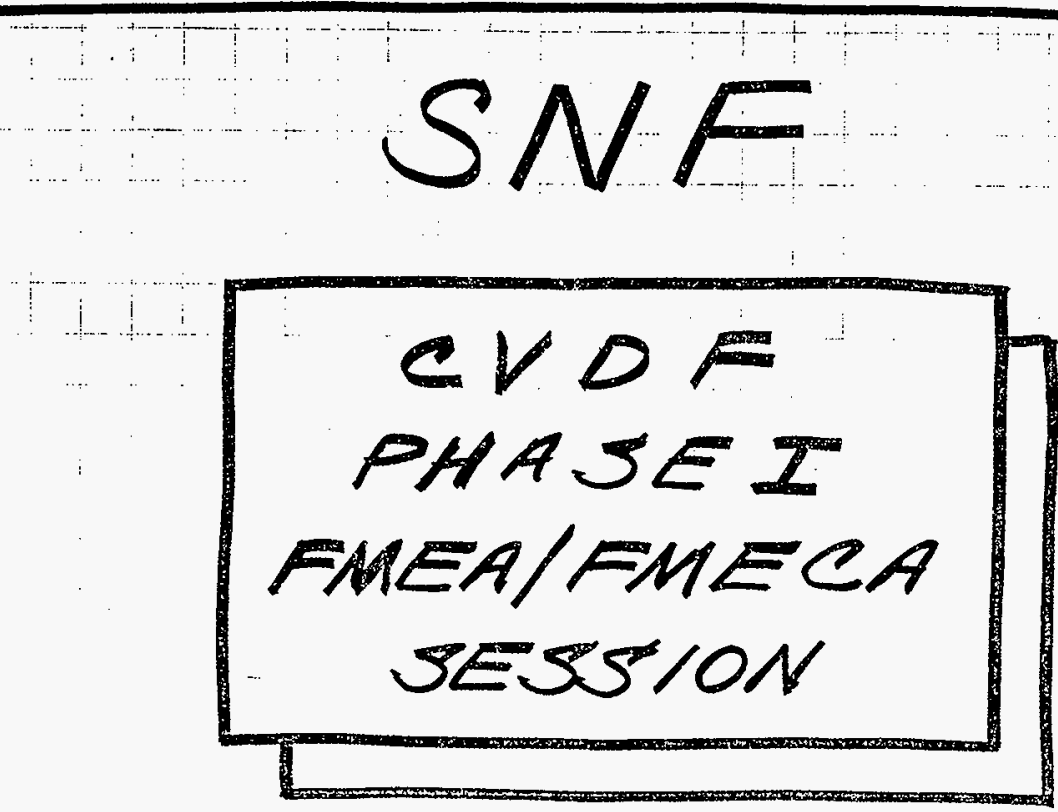

AUGUST 18-21, 1997

$345 \mathrm{HI} \angle \mathrm{B}$, POOMI 124

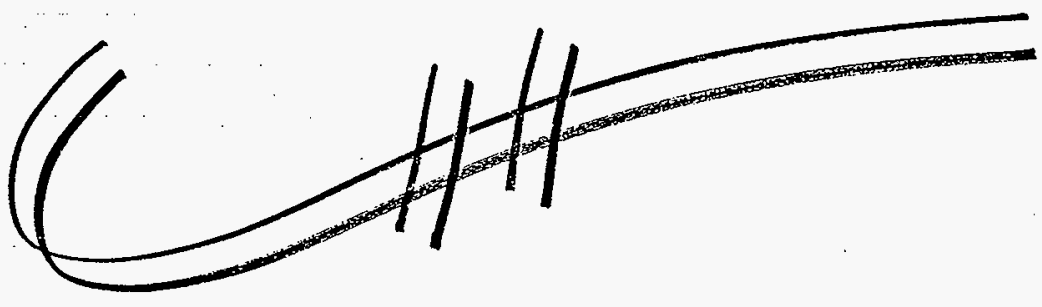


Aetion Itens

(2)

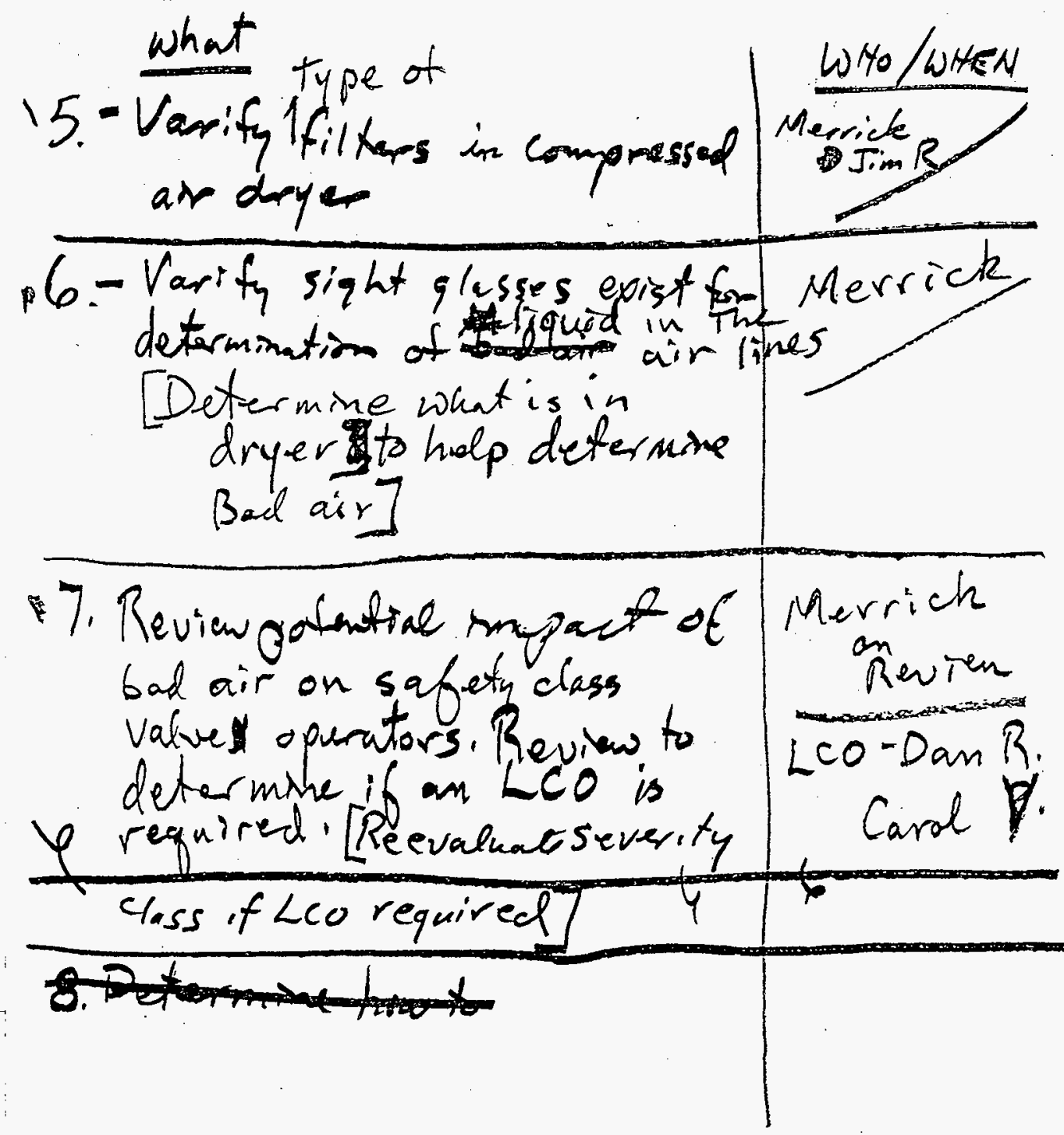


(3)

WHAT

WHOLWIEN

象. DETERWNE MINIMUUN He JOHNI. PURITY REQATT.

9. DEVEDPMETHOD TO VEVIFY TOW $Z$. He PURITY@STE RECETPT IfOR ARE.

10. Switch to service John I. water to supply or water (Design Change)

* 11. Determine mpact of glycol on MGO develup recorery glon if required; and

John Liwin fallure detection methol if required

12. DeveOP RecrieY PROCEXIRE(S) KALT A. TO AUROHODATE DERAS THAT GEOGE V. WOLATE TECHNIEAC SPECIFTCATOUS. 
ACTION ITEMS

WHAT

(4)

13-Varify function and Merrick need for Dam ger DMP-8060

14 - Recusal nat the approach

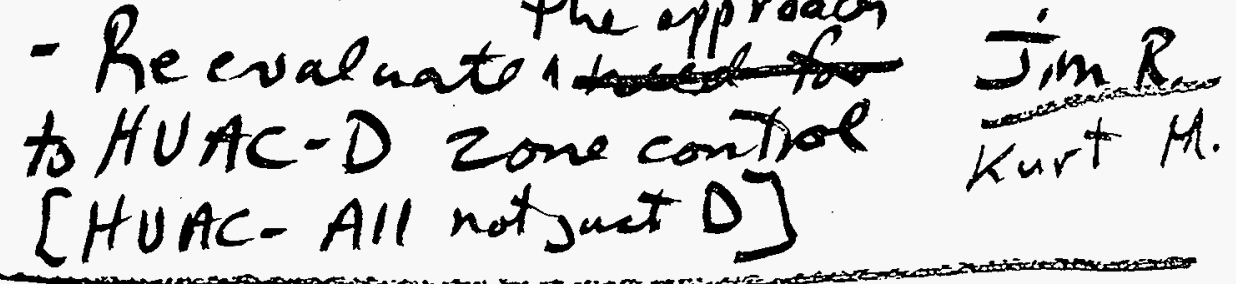

\$15-Upqrade 2HUAC Drawings $\operatorname{Jim} R$. to show a condensate . Kurt M. drain lines and seal pots

16- Upgrade HUAC $P\left\{F D_{S}\right.$

Show: 1-Correct conn Jim R. of Tank 4001 to $\mathrm{Kurt}$

- local exhaust sis

2. Covet connection of process skid vent to lo. el exhaust system

3. Show the isolation damper in the process vent system at the bay walls 


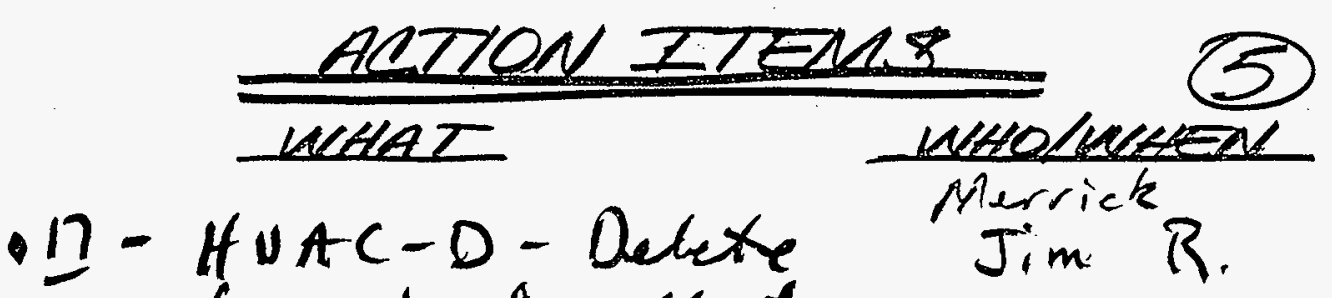
fume hood callous

18- Va if Merrick Outside air damperintion kurt $M$. general exhaust ar flow

19 - Evaluate the need to provide a" vacuum "b peak in The

$\operatorname{Jim} R$ :

Torn event of Loss of AN to HUAC-B

(Design a change if required) [Safe exit thru Door

20 Evaluate need for local bay repeater alarms (from problems Whet $A$. in other bays)

- Il Determine needs of programing Dick W. for Mes

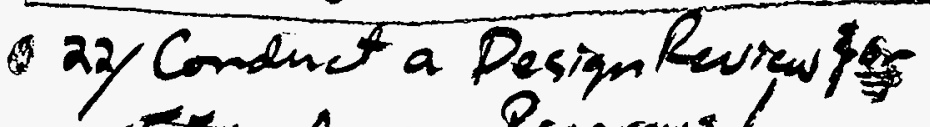
Fete on Programs

Dick $\omega$.

NF 2576 Row O 
ACTION ITEMS

(6)

WHAT

Who/WHEN

23

-23) Do a dosestudy on the Cavol P. PUC, Determine : 6 desian requires change. Generate DRD or Desisn change as required.

-24/ Develag procedures to use in stalked bach no pumps John I. $D$

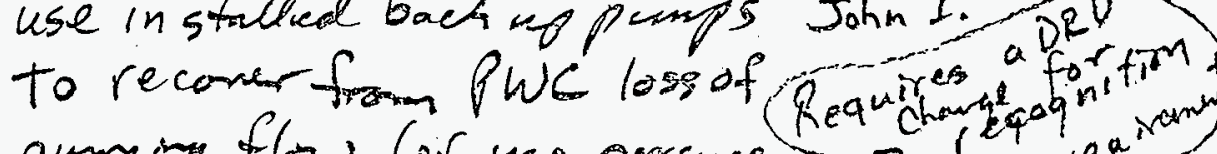

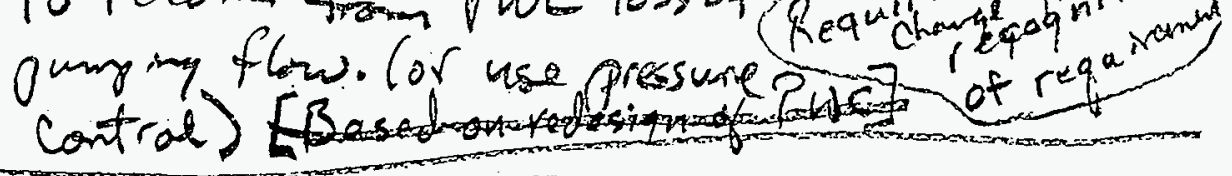

-25/ Recunluate Sowase Term for Jeht of. Pwe and thermine if DRD [Include woher safety changus are required. [DRD to and ALARA]

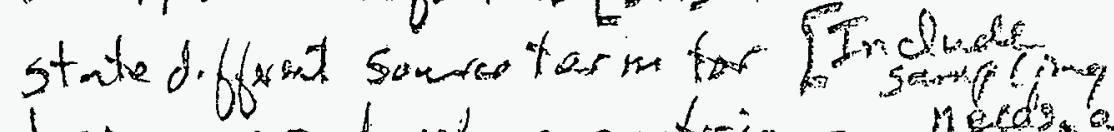

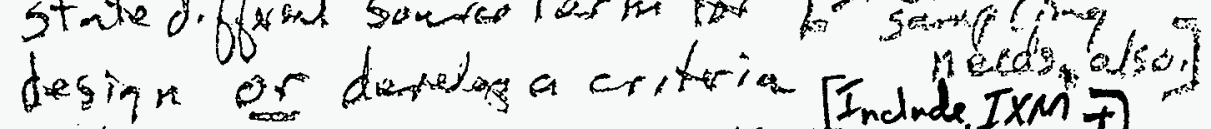
that resuldes in a designe thex replacement] provides an opeaber pisco AT Hibotianes? 
Action Trterns

(2)

what

Wotho/when

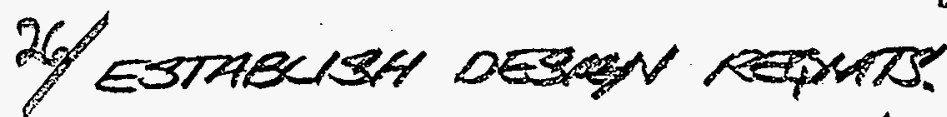

John I TO MITIGATE LEAKS ON THE PWE SWBSE

27/ Develos a procedure for checking John I. the inner seal of the mcol cask cunnulas seal

28/Determine sevenity class to John I. the "caste low water lenel" for Tw

'2V/(ENBURE FAMCKE TO RRAIA DCKN.

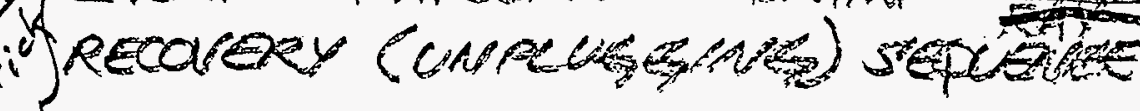

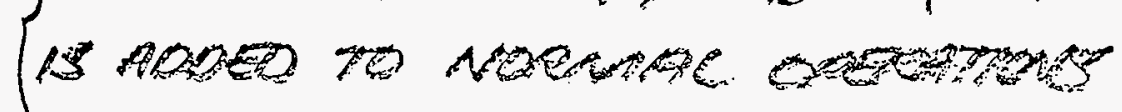
exocerute.

- enamamas hao lecu lefer

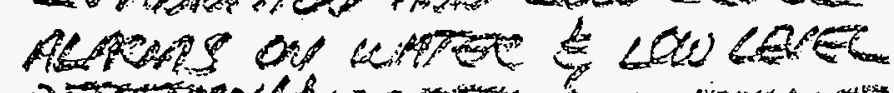

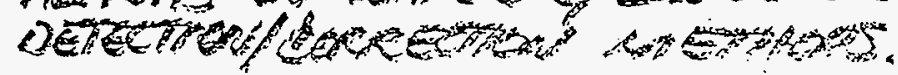


Action Jotems

(8)

30/ Evaluate need for $\frac{\text { What }}{\sqrt{2 h} \text { h I. }}$ RGA

.31/Review EPD for faclure John I. mode revovery proudures

32. STATUS \& CONICETE DRD CHANGES (EHHT TOHNT. TOTAL).

39. जTATHS F COMPLETE CAROLP. DESHN CHANES $1-8$

34. DEVEDO NETHOD TO KED VOW I. IXM FEOM GOING TRU

35. ZDEMTIFY NEW STRSBSY FOE COMBNOO HVAC DEBMN, CAROCP.

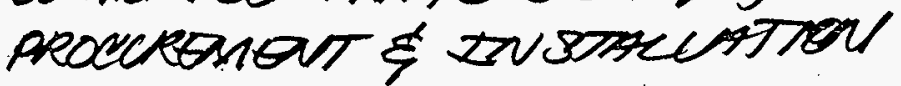
FOR SINSLE OVERSISITT. 

Pupposef Phose I

Sereen 5ystems, Sussystems to determine which will be signeficant cantrabators to plant down tims. 
Voting for Phase 2 FteAECA

1. Is system alyaificant

to plont downtime

- Failune Rate - Freg g Occur.

- Restoraxion Ruti-Mreantome to restore

2. The "yes" desis notimn salicates that the followen purewers will use the tubulated information to more precizely selecte the components to de a eltaited FMECA. 


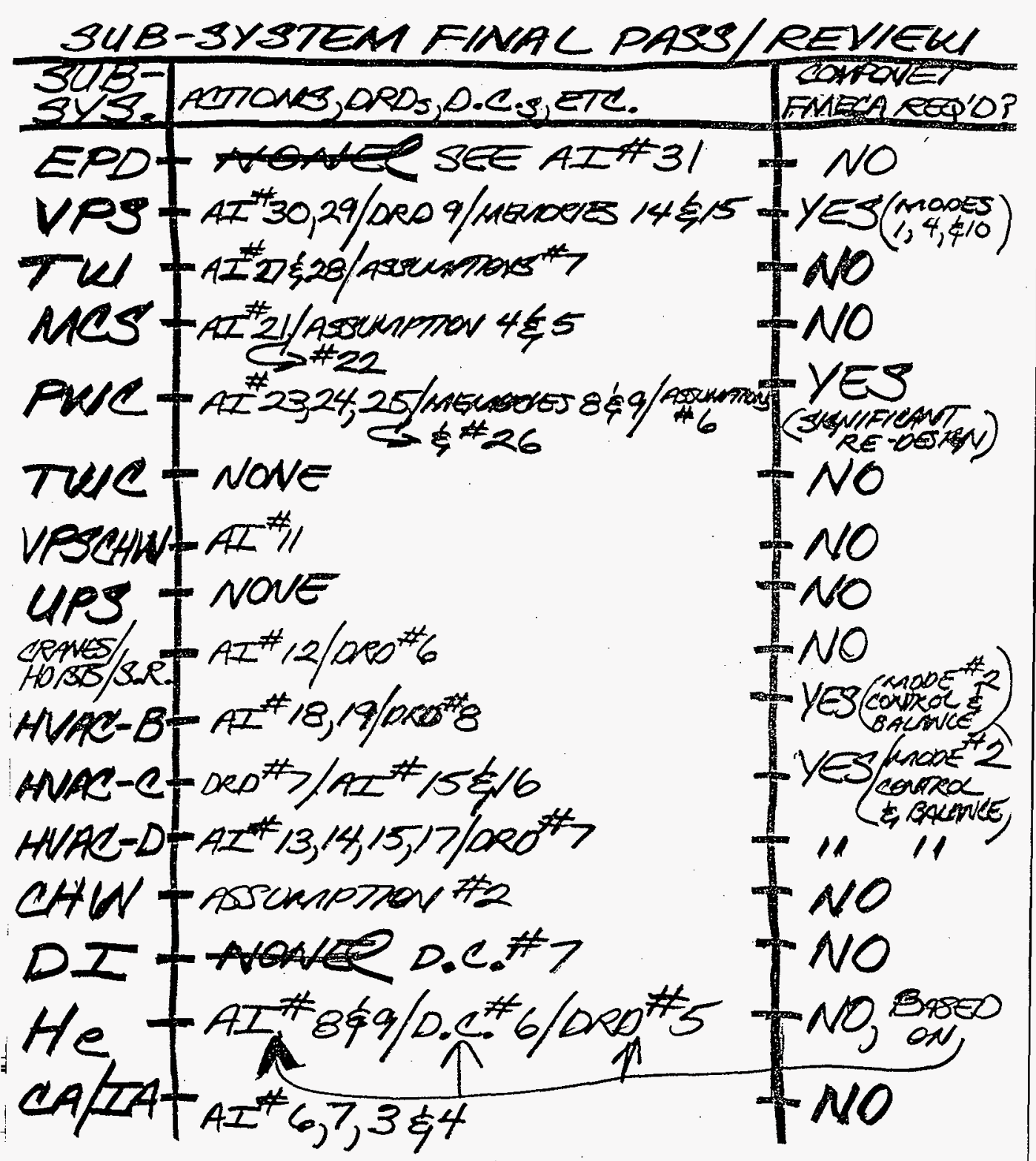


MEMOALES

(1)

1 - Iduntify source of defonitions for Severity Class-Giva johnd

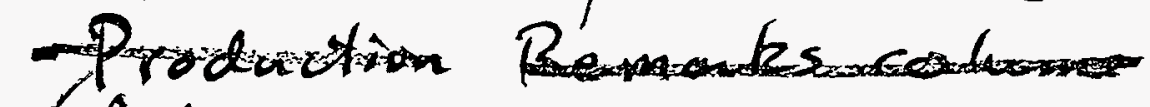

1 fots

2.- Remanks columat is for darifiemation/Aation thase

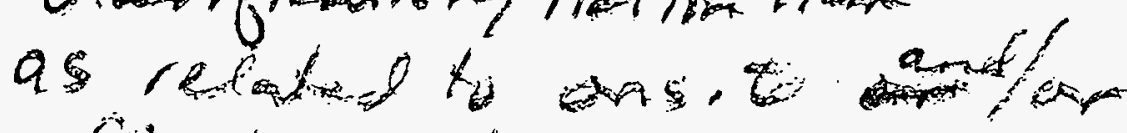
off $s$, to

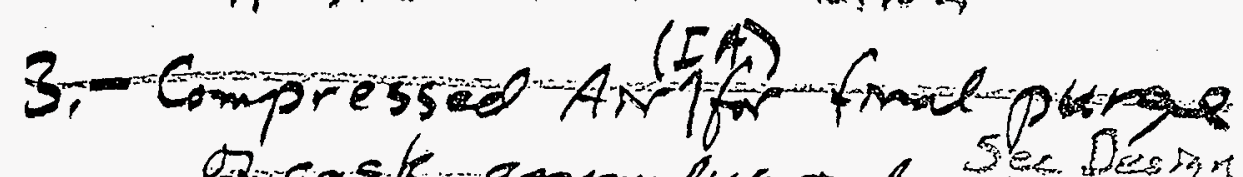
of caste annulus anc Sec Desizn

4. - Varify that thermo analys is is being done_Caral V. verify Dons

5.- Will Address recoury, recarery fime,

Crisk after completing entre review

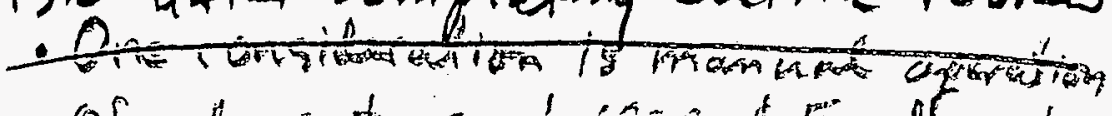

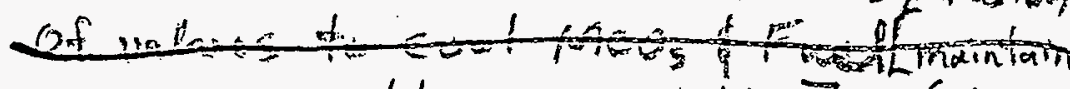

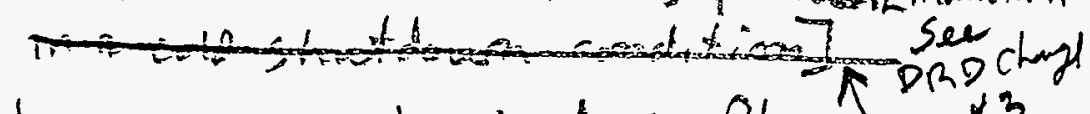
$\rightarrow$ To be accomplished in Phase 2 "3 
MEMORIES

(2)

6. Evaluate for Lessons Learned from WESF HUAC Design \& Controls

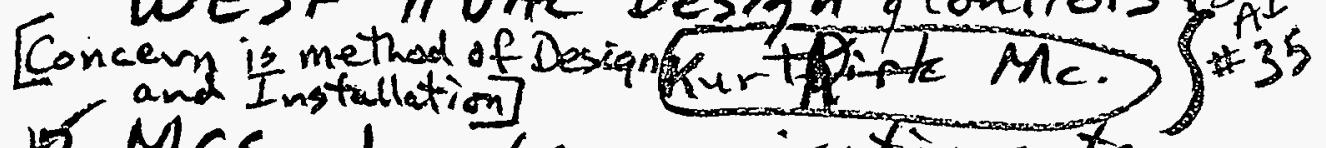

5. MCS- Loss of communications to control coom assumes that communications has exceeded the programmes time out

- Consider remote replacement for Pule Recite Pump- AI\#24

a. Any process, belong resmbets in fuel corrosion \& corrosion products

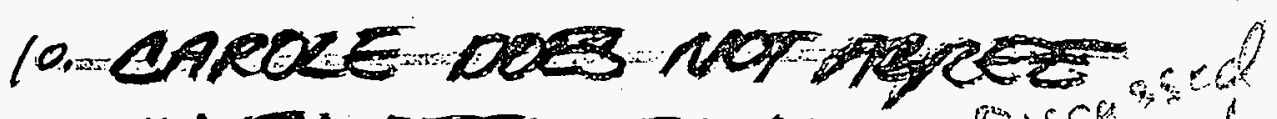
WITH DEFWPRTS.

Wonetech sheets for consistency of determination of severity class [is it with mitigation or with out?] [With mitigation!! ] 
Memories

(3)

12. The oppose of this Ph I FAEATFECA to to focmson

2. Eons silerectione name of session to Coded FiE MA

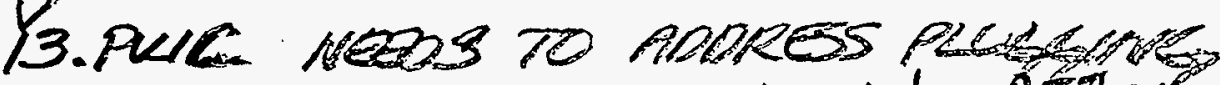
OF FCOU HE TER. - Merrick. AI 36

14. - Vacuum pump and system being Q evaluated (May result on changed component failure rate) - Phase 2

15. Operating with out the blower (in helPS) *31 is not abnormal (Procedures will be developed (2) to operate with or without blow)

the. 
AsзиметLON

Li Service wester (nat potable water) supplies DI system

2 LOSS OF HVAC COOLIES DOE 3 NOT SHUT DOWN PROCESS

3 HUAC will be stand alone with interfaces to MCS $-5 e 0$ DR $\#$ ?

$.4)^{1}$ We can program out put signals for failure positions (Assume correct programing)

-5) We will test for all normal sequences $\$$ selected off normal events (key is selection of off norman)

"6) Hiftagher source Term (by 1000 Times) than was given to Merrick for design 
- If Ion exchumptons and related instrumentation (2) THC system and replaced with by pass flow control for pump protection and pressure control. Duplex strainer deleted. Tank filter (PV-F- $3^{*} 15$ ) and related instrumentation removed. System now has safety class temperature shut down. Valve 3*15 deleted and TW ${ }^{5}$ disconnected frow Pw (manual, installed jumper a vailiable for interface as needed).

8. WILL NEVER UBE THE RECVCLE WITHOUT THE CONDENSER.

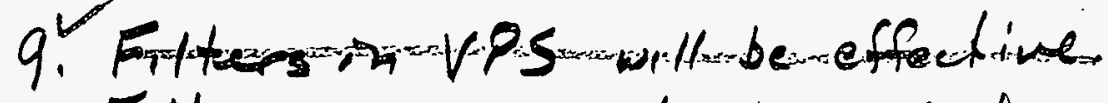
Filter on MCO is nat plugged when received at CVD. 
(1)

1- Investigate maximum allowable connection pressure in cask (Quick disconnects attached to cask) Andy Kerr /John I.

2- Operating Sequence -close Mao Block vale - when??

Identify Timing Dick 6 . Dan Re

3- Potential Design Change-Chea vale in air line to seel ring Merrick

H. On Owe H-1-82222-Add loopsig Service to $R \mathrm{~m} 132$; check if 25 psis Service is needed Marrietef John $t$

a. - Verify alarm type (PAL 5008) change as required [Need high $\$$ Low Pressure] Dirk wo.

- 5 Potential Design Change -Allow the use of IA for formal purge of cast 
DESILN CHANLES

6 ADD PT OR SWITCH UPSTREAM (2) OF He PRESSURE RESULATUC TO DETECT He sUPAY PRESSURE Mierrick DDick wi Qupdrty

- $z$ Global Change-review all $P \& I D_{s}$ for change from PAL to $P I$, Mikeride

8) 82164 - change

$$
\begin{aligned}
& \text { Mer to edr } \\
& \text { Merrick/Dick } /(E D R) \\
& \text { MJR) }
\end{aligned}
$$


DRe chanes 1- \$Propect philosophy on spare.
parts

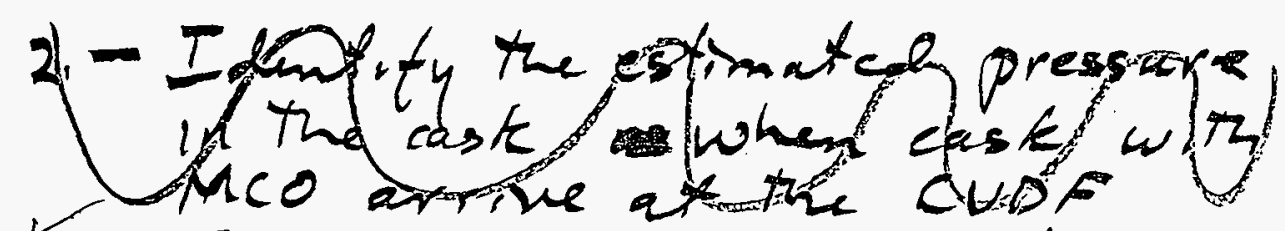

3. - In clude cagability to putherco in a colg shutdoun condition John

4 INVESTIGATE TIE DEYNESS CRTBUA ON INBTREMENT TO DEREPHINE REQUIRED DOW POINT FOR THE CASK ANNULUS AREA. H

'5 ADD "CONTINOUS BuppLY OF He" REQUIREMENT:

- ensures ovoepressure de coss of

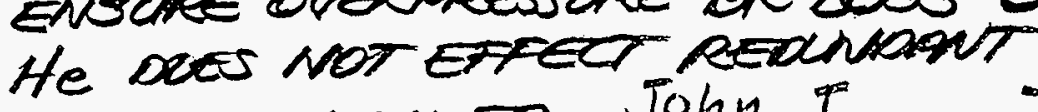

- scrply treallete. John I. Johng

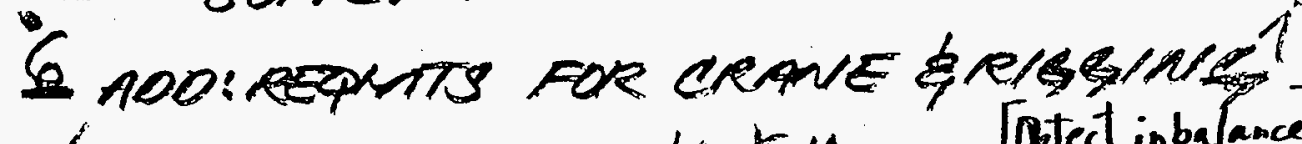

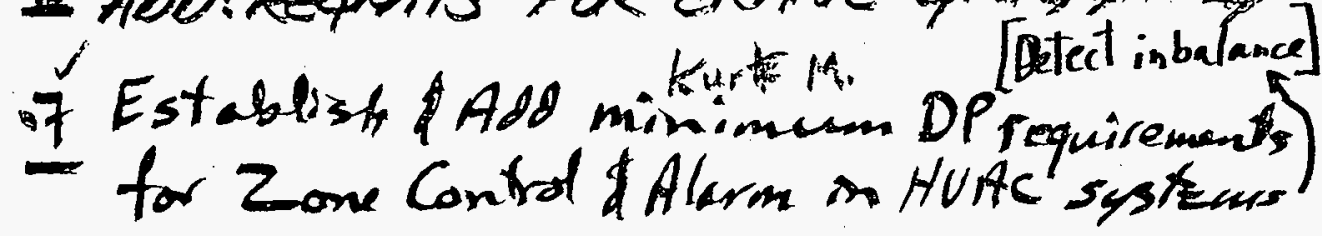


DR D Changes

(2)

- 8- Potential Change. Require a stand alone AUAC control System with interfaces to

$\checkmark M C S[H V A C-B]$ g Dick $w$.

- q- Identify require mint for Moo block values with respect to particulate in water streams John I 


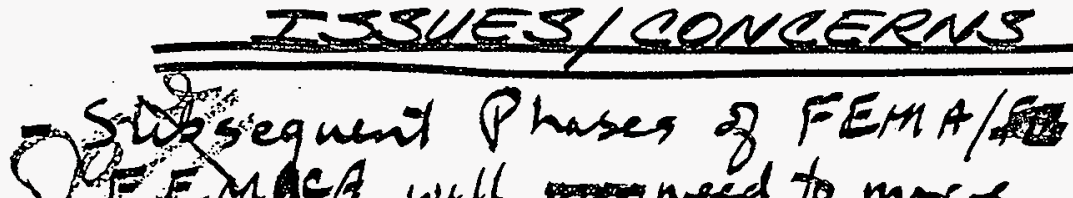

(1) FiEMAla will need to mors fiqorously iduntify componet fallure rates Algha, -

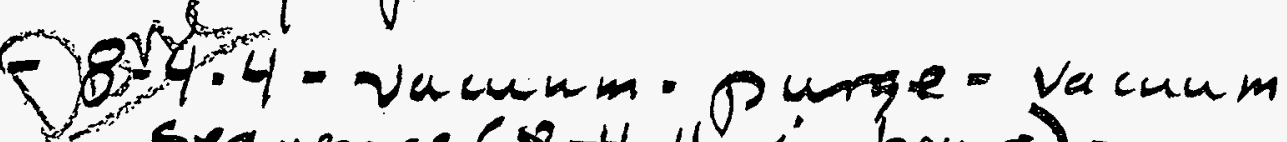
sequence $(8-4-4$ in hours $)$.

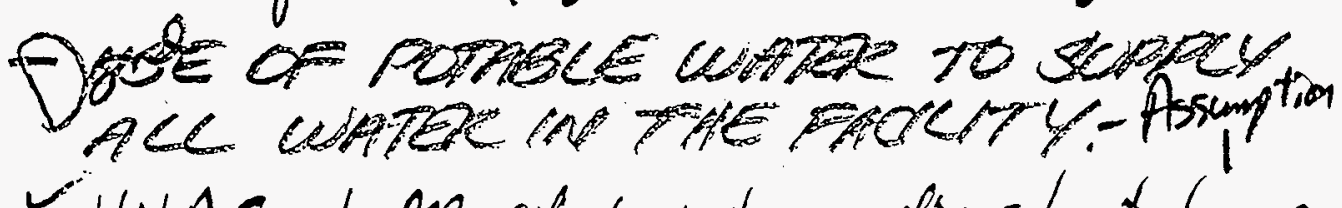

$\simeq H V A C-1$, Multiple inter actions/miterfaces

ib. Acomplow system

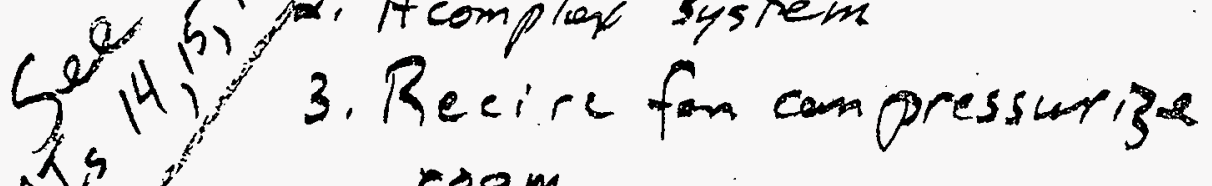
room

4. What syshem controls HIAC

$y^{2 x}$ (Mcs todute but!?)

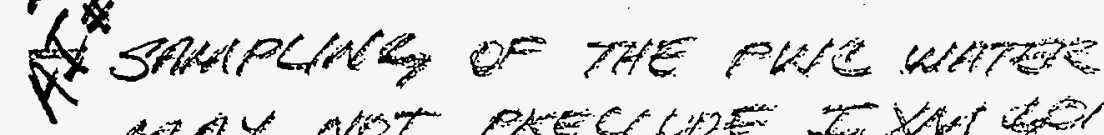

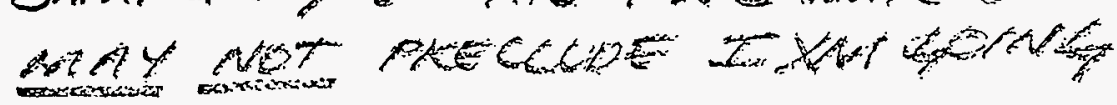

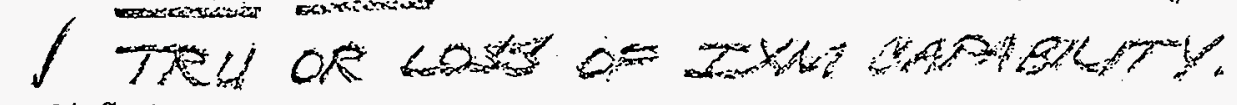

DED

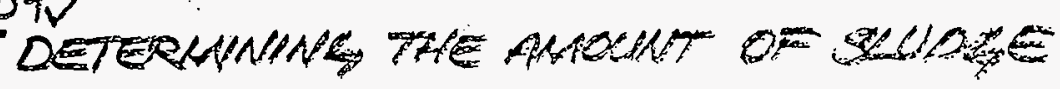


DEFINITIONS

- Component Failure Rat, EL: Extremely low - not in life of facility

$L$ : Low - maybe once during design life

$M$ : Medium- I diary lifecto l per year

$$
\mathrm{H} \text { : litigh }->\text { I per year }
$$

- Failure Mod - Subsystem

- Loss of to - Local Effects - what does it locally

- End Effects - end effect as result of failure

NF 2576 ReNO 
DEEINITIONS

- Mitigation of Cause.

(2)

- Prevention fentures (- lessen seventy
gconsequences

- Reliability of Mitigation

Some fow defonitions

for feulures rotes in

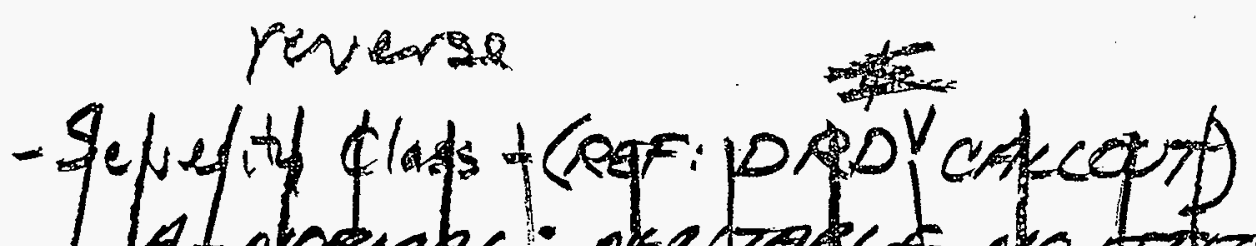

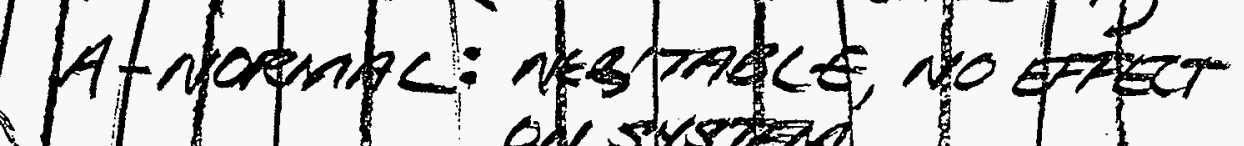
on system.

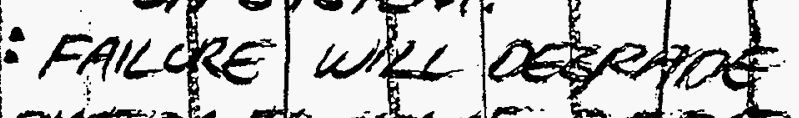

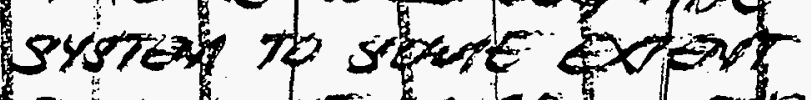
Got wiL wor dnuse lun nade

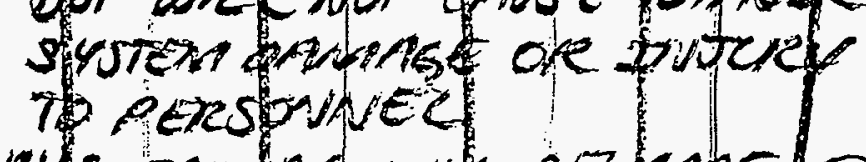

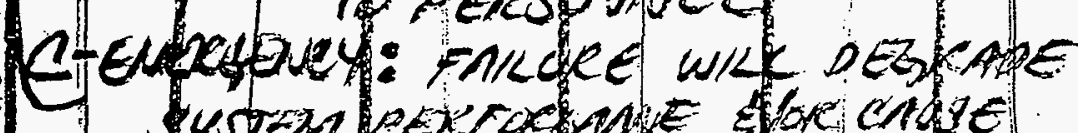

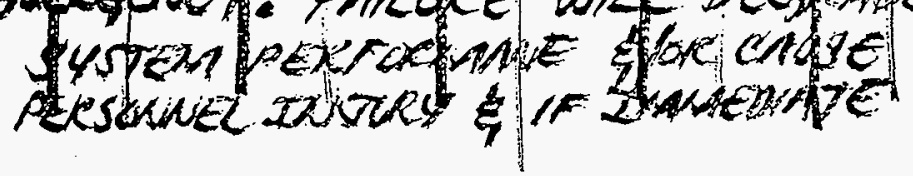


DEEINTIONS

(3)

- SEVERTY CLASS (KEF: DED

A-NORUAL: NEQSGABLE, NO ETFCT ON SYSTEM

B-UPSET: FAILURE WILL DEGRADE SHSTEM TO SOUE EXTENT BUT WIL NOT CAUSE MATOR SYSTEM DAMALE OR INOURY TO PERSONNEL.

C-EMERBEVVY: FAILURE WILL DEGRAE SYSTEW' PERFORUANLE $\$$ /OR CAUSE PERSONWEL ZWUURY, AND DF IWMEOATE ACTTON IS NOT TAKEN SERIOUS LWUTRIES OR DEATAS TO PESSONUEL SIOR LOSS OF SYSTEA UUILC OCCUR.

D-ERULTED: FAILURE WILL PRODUE SEVERE SYSTEM DEQRAOATIOS CAUSINAS LOS3 of SISTTEM EloR

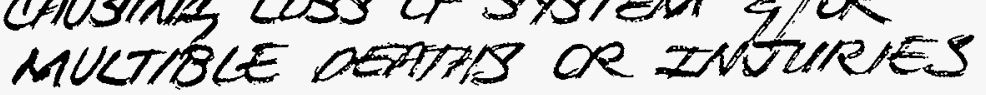

I NOTE: LEVELA, DOES NOT REAUVE REPAIR LEVELB, IS MINOR REPAIR. LEVELC, SIGNIFUAANT REPAIR $\angle E V E L D, B E Y O N D$ REPAIR 
SUB-SVSTEM BOUMDARIES

- CALTA

(1)

- cA conpresode, TANK, \& OXVYeR 100 :PSIS FOR CA \& 100/25 FER IAA (25 TO HVAC, 100 TO PROCES8) LOAO3: HUAC DAMPORS, \&40V'

SAS OARATEO VALVES ON THE PROCESS BNOE. CA 10 SCAK RING CASK PURE.

-HE -82222 Sht

Leased twke trailer to Mcolincluding inside meo, eacabay)

Bothles - 乞 1sropsin $\rightarrow$ 100psis $\rightarrow$ 20psis

Punge ong

He Quality>99,9\% as delivereal to Meo 
OMII 9LSE $A N H$

$\therefore$

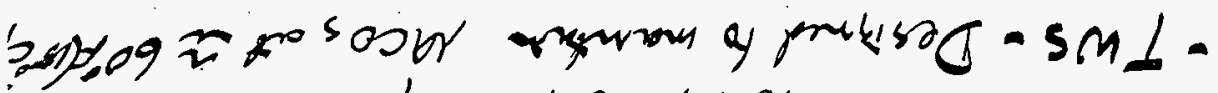

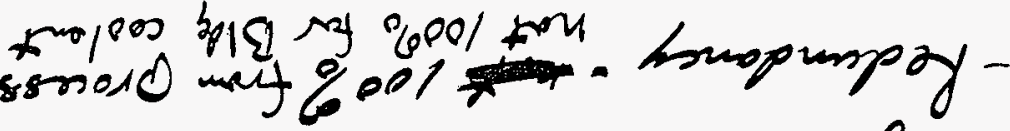

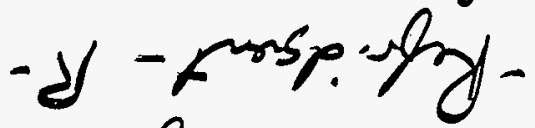

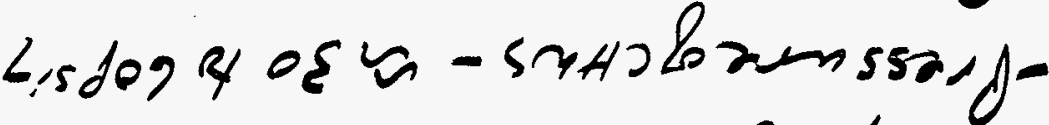

$$
\text { sosh-oh }-5 m+10 \text { \& dmal- }
$$

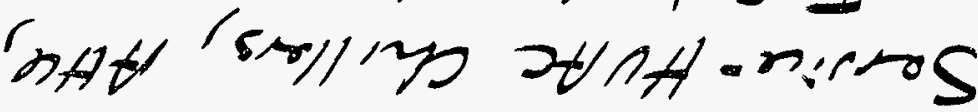

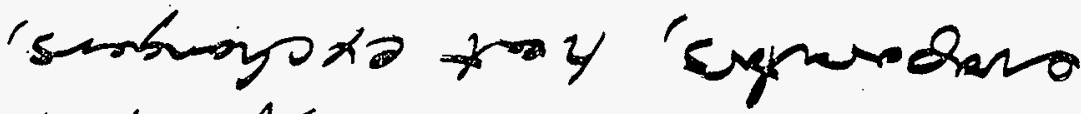

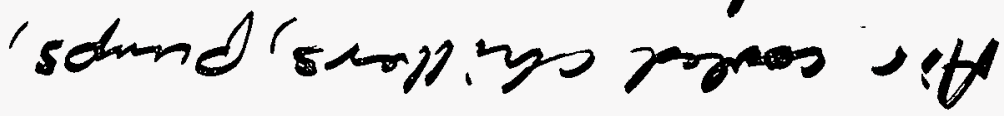

$$
\text { 8orr \&-'102e8-1-4 } 77420
$$

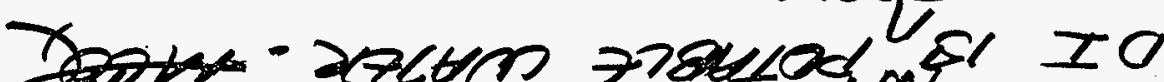
$\log \frac{6}{6}$

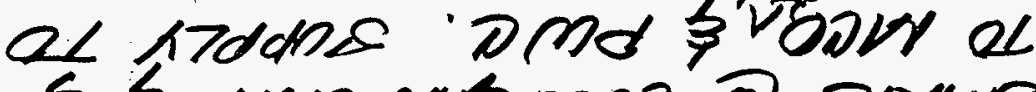

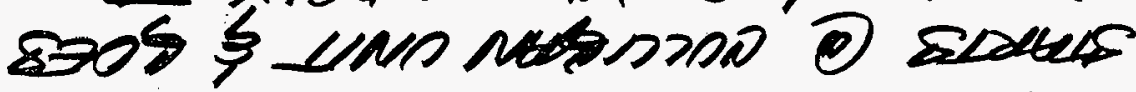

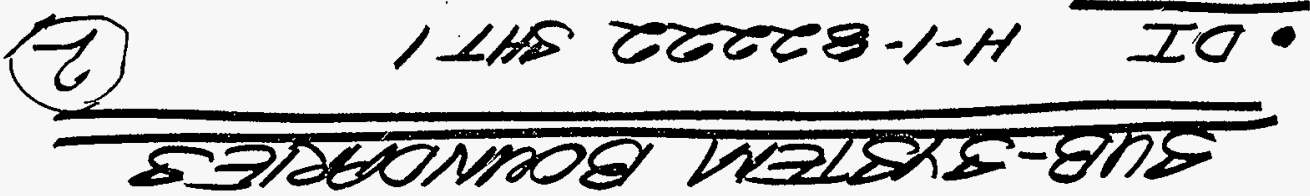


3UB-3USTEM BOUNDARIES (3)

- VP3CHW H-1-82224 CLOSEO LOOP, ZNUTRRACE WITH conbenGER \& HEAT EXCHANBDOS

- true

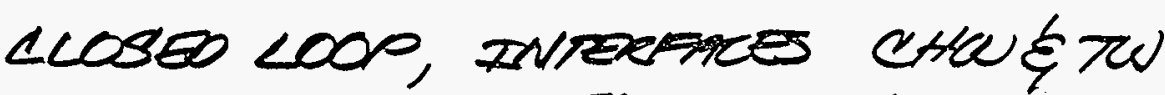
LOOLEO BY CHW, THEN COOLS TU. REDUNDENT PEMPS

- HVAC-C - Process Bay Locale thaust - hoods and process unts Thre the stack H-1-82206 
Sub System Boundaries

(4)

UPS - H-1-82247 Sat 3

- 8 hrs continuous ops

- Cringing

- Oz Monitors

- All of Mes

- Safer class I kC

225 Amp

$47, x$ kun

$120 / 208$ volts $3 \phi[$ no 208 Loads]

HVAC-B - Bay Recire-Separaco

for each bay -

$1-H=82204 \cdot 6430.19$ for energy Conservation

Inter locks w. th exhaust system \& Fire protection 
Subsystem Boundries

*Cranes, thists, seismic restraints

- 2 Ton Onerherat in each process bay

- Fatable crane for Ix mo memut

- One sersmic restraint per neo care troms porther/bay

- HVAC-D H-1-82206 PROLESS GEVORAC SUPACY ETHACBT tic Frantarenes

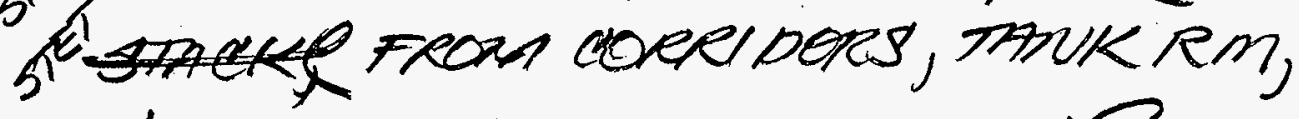
G WECH. RM. W 
MCS - H-1-82291 Two enrol (6) Computer systems - Desk Top -Bis Screen windows NT, Graphic representation $o$ rooms of systems; Printing capabilitison any Ooze; Can develop any report for statue of Tracking, control $3^{\text {rd }}$ Engr Wort station-Develogment $\$$ on line changes to program. $4^{\text {th }}$. readowly in safety office Alarms - Control Rm -computer act nolleger -

- For Rm -Rod alarms only Mo Alarms in bays Status light for work in bays Control Rooms manned during any bay A person in ane bay well nat thaw of problems PLC's in other bays miles not fred by control 
MOS

(2)

PLC is networked to MCS

Programing oren in $P L C$, 2 P LC, - normal uninterrupted back un

7 If cobrates

Failures can happen on section of card, a card, a carinate, a room

Each rack e hae a small buss with 2 communication. * Dual communications with control rom but ace IOs are singh point

PLC, very reliable; Single point failure is $L$ EL failure rate (bot of faith in PLC)

Gand watch dog-exrst but not sure if it causes fail position or maintain last position 
SUB-3VSTEM BOUNARES (3)

- Fur H-1-82223 $4-1-82164$ trof

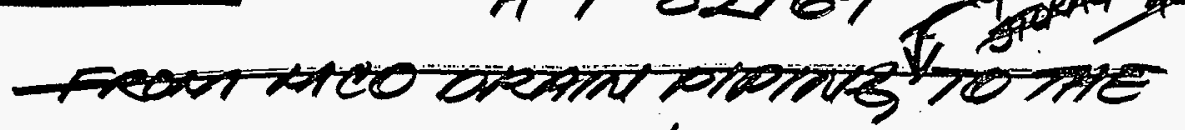

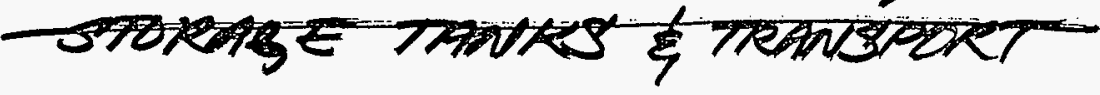
TAANEE

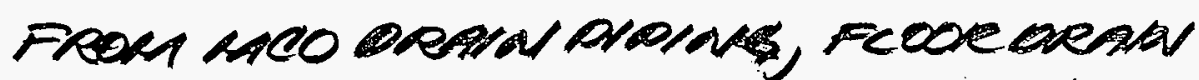

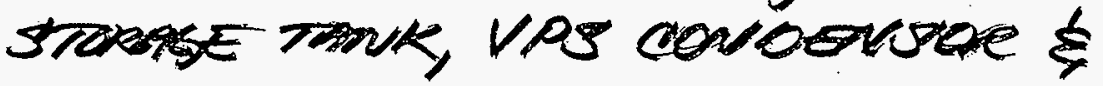
TWIS TO THE STOBAE TANK \& RECEVE TANKS THEV

$\Rightarrow$ THEV 70 TTNISPOCT THUKER

-TKL H-1-8216/1582163 CIRCULATES CLEAEHWATEE TO THE LPM CASK ANNULUB, WATOE 10 HOT OR

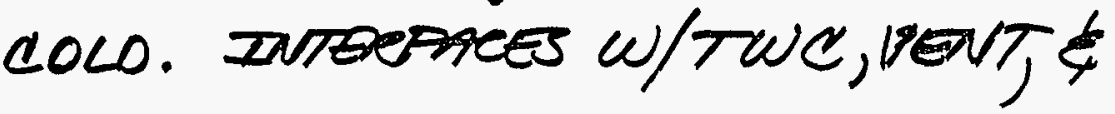
DI WATER SYSTEM. A CRITICAC INTERAATE IS WITH S.C. ISC SYSTEM, ADOMTIAL ZUTERARES WTHI He \& ZNOT. AIR. 


\section{SUB-SWSTEM BOUNDARES}

\section{-EPD H-1-822417082248}

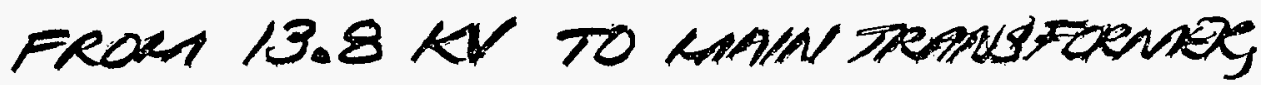

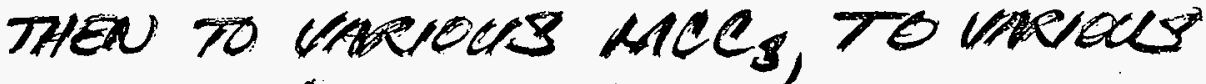

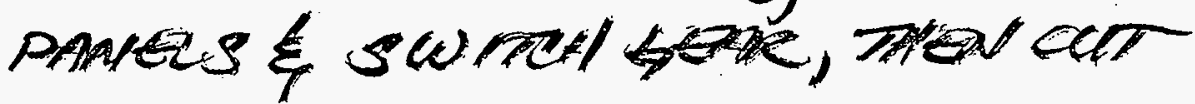
10 VARETS mpontents.

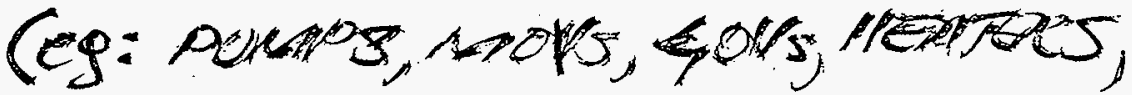

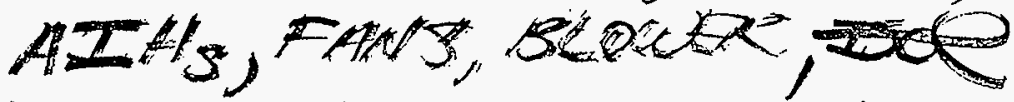

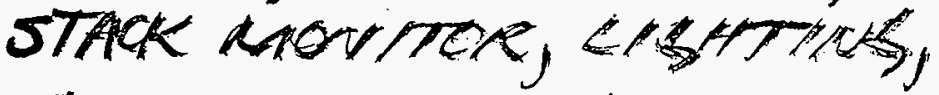
CAMCER, ALAKRSS, KITHIEN APRLARNAES, COMPRESSENS, CPANES \& HONSTS, ETC.) 


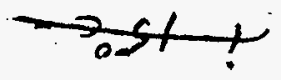

compuns " "SAng "'

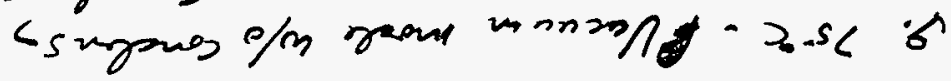

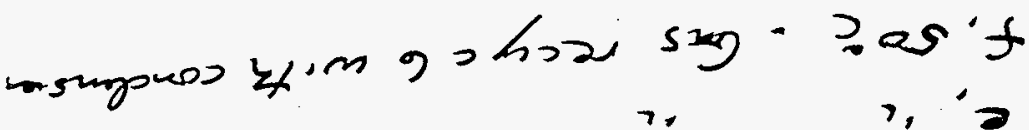
rugars firstin

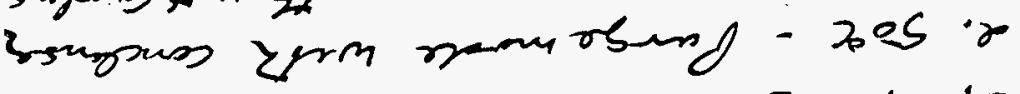
" froyim " $"$ " 1,0

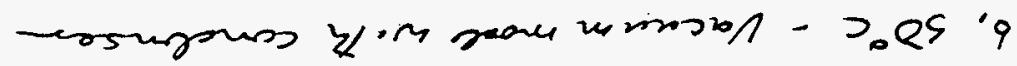
reand yom unaye spom - DaOf ib

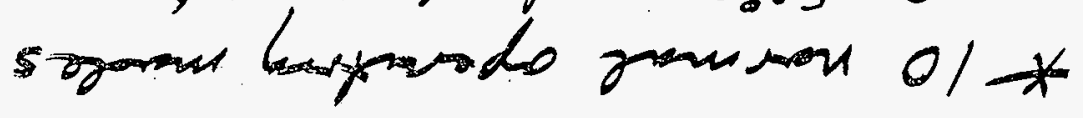

(b)

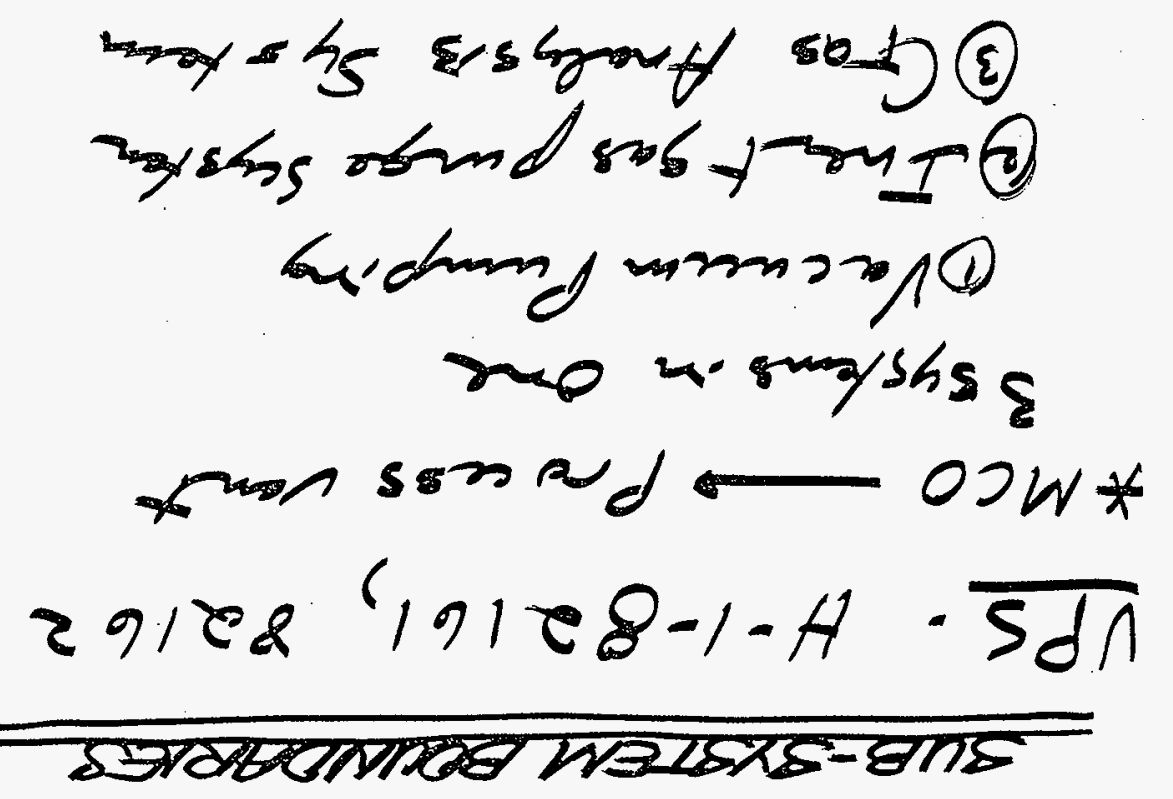


VRS SUBSISTEM

i mazoe

i conponerts

VACcrem peutP

BLOWE

conpeasae

VALVES-1,

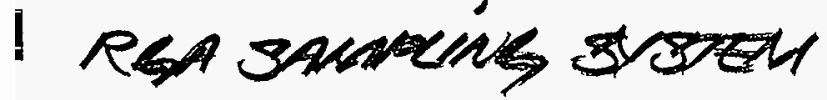

1 Incspunarnatron

I RECYCLE HEATER

I DRAIN TAONK

1 FILTER (FI*

FLEX HOSES (WTEH.)

MCO HEPA FILTER

MCO SERLS:

MCO VALVES 
Spues froy"d rean rbeng $-11 \%$

sous zim roul ofung - "X

"spung froy'm ". ." ?

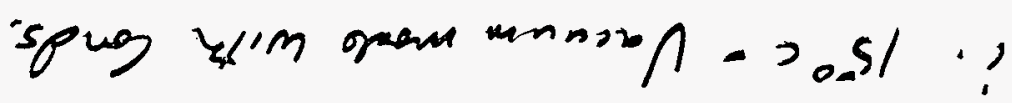


Opening Remarks

Must build something Tan works

Thru entire life ye 6

Think reconery time/ process"

what if it breaks, fails, f $_{c}$ -

What must be done recover

- Procedures - Desiznchanges

- Spare parts

Process Emphasis.

-(This Process -This session) "What if?"

What recovery procedures do we have?

t ave some but this process will help identify needs 
Ogenseg Remake

- Some components house a life time that exceeds the ne life of the System

- As a team we need ito bo thundering probability

- Philosophy on spare parts - Site ste.

- Using the Severity class identification the project will finish procedures to address the 4 conditions of operations - norman, upset, emergency, faulted

- Budget attucks. $1^{\text {st }}$ Contingency $2^{\text {rd }}$ Spare Parts

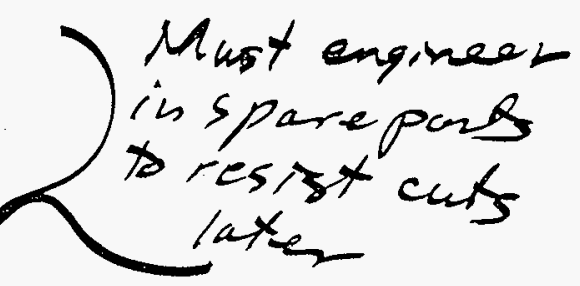

H NF 2576 RenO 
CVDFunlity, OhaseI FMEA/FMECA Sessiz $8 / 18 / 97$

Nare

c

Qhan

ansint

$=$ hand Atfarringtom

LMHC.

$376-2331$

$1+8-71$

E(Jeff) Parker

ARES Cory

DESH

$946-3300$

$\eta \bar{v}$. Wiemers

Menaick

376.9516

Jave Mungen

Memick

$505 / 662-0606$

Jim Robinoon

DESH

$783 \cdot 7404$

arol Pittoff

Jalt alacinis

DESAI

3765655

Kay Lavow

37.6-9390

$<J \operatorname{lm} \leqq$ CRacKEN

373-1698 Q3-85

Zek Whttelerert

FDNW

$373-6653$

Procle vincens

Desit (teday)

$376=4663$

$23-86$

Danie) A. Reny

NHC

$373-1513$

$R 3-86$

John Irwin

Jasan Assoc.

$373-7518$

$376-3619 \quad R_{3}-86$

75

HNF 2576 


\section{acon $a$ cse $\exists N H$}

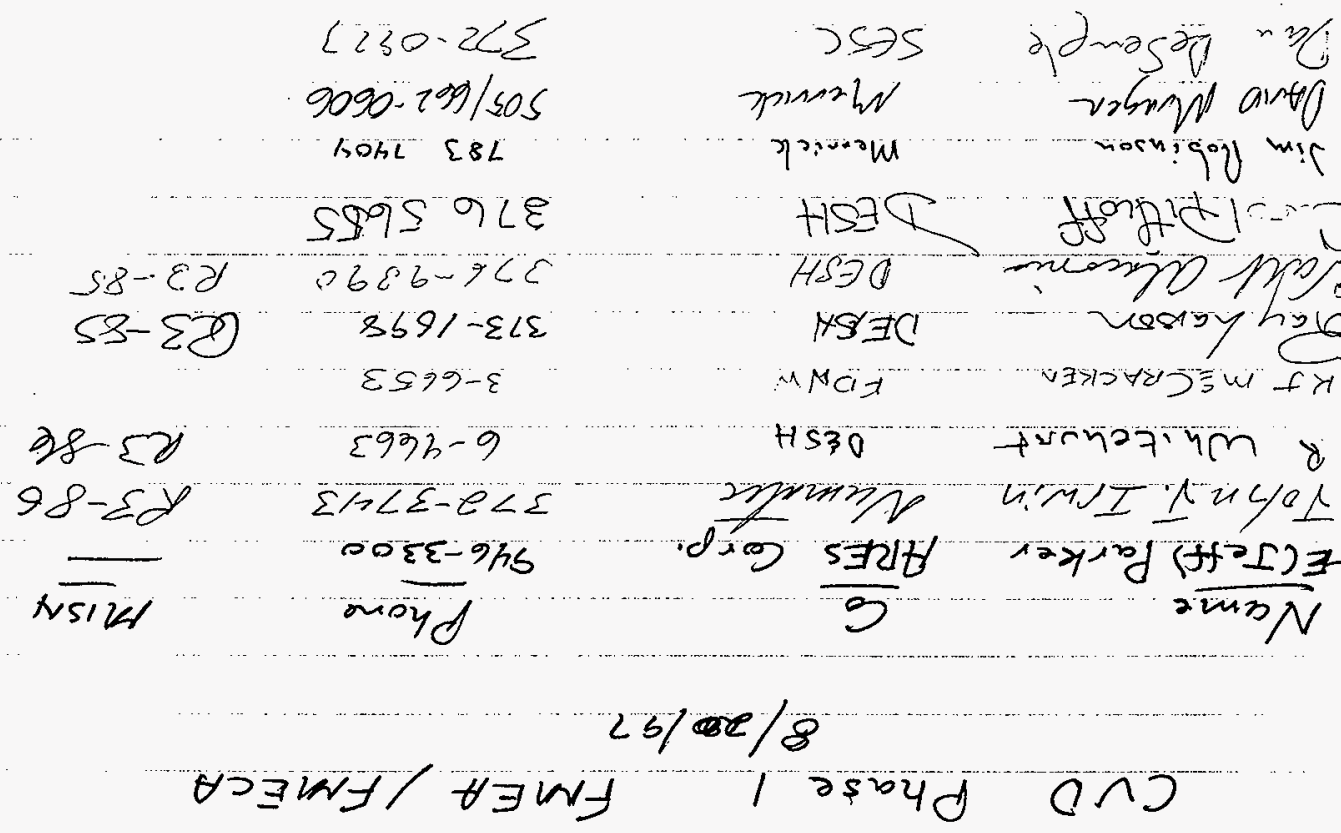


O min acse twht

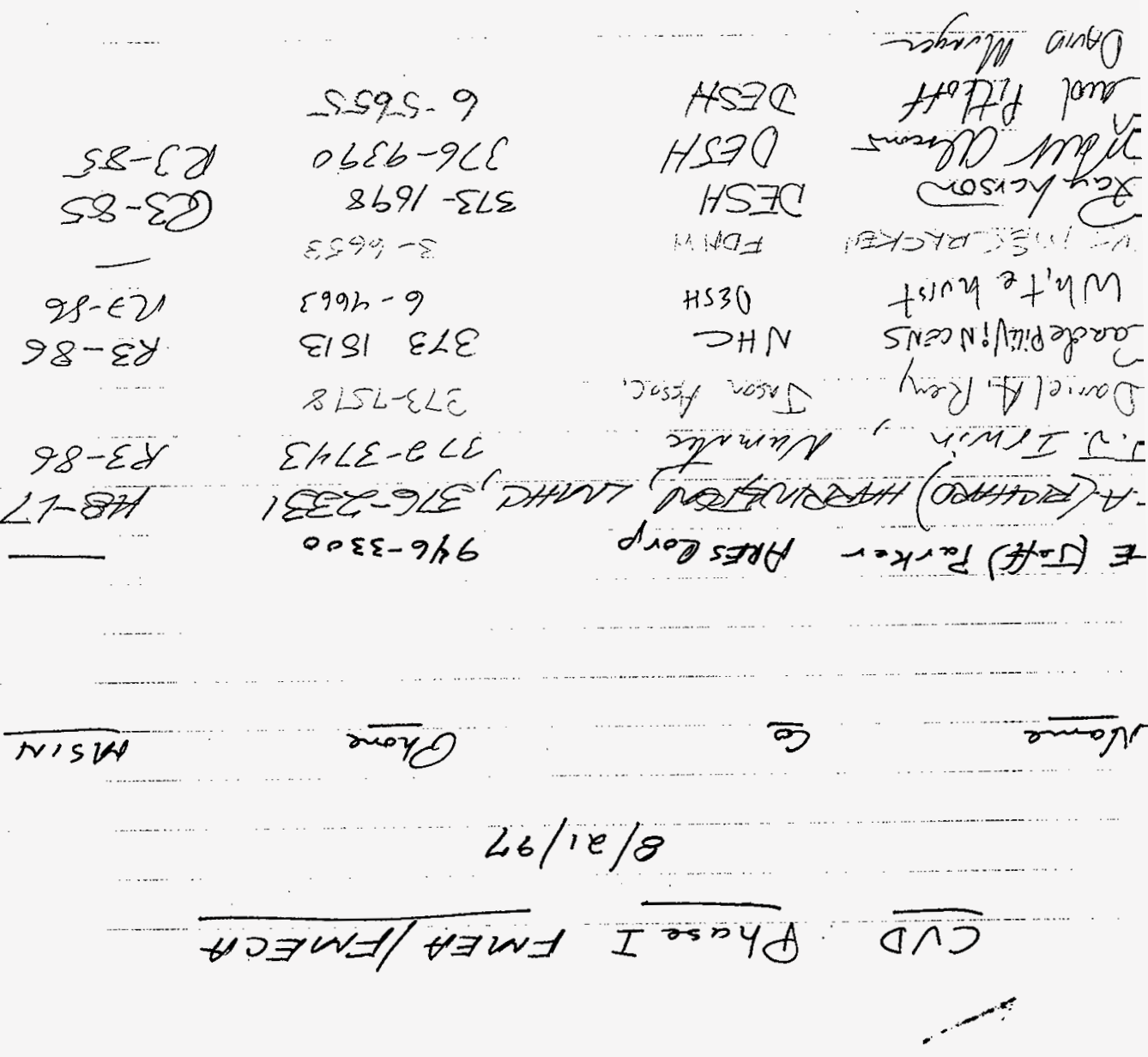


GUIDELNES S EXVECTATIONS

- OPEN HONEST COMUUNICATIONO - ALTIVE LOJ ENINE EONIDEATION

- zDENTIFY Fale vo pepceotions

- hazop vs fena vs femea - PECOSNIZE UNIAUENESS OF EAM - VET, ALLTHREE ARE RELATIVE

-TYPICALFEM/FMERA DONE@THE conponet LeVEL, w. BVBEen - OUR FOCUS (PHASE) - ANALYZE FALURE EAFET OFA OUESAL SW TSEM

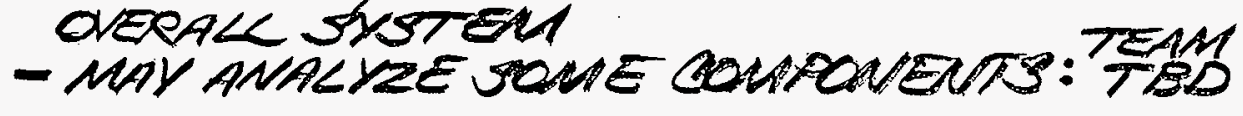

- roles: Faclutatcos \& yol

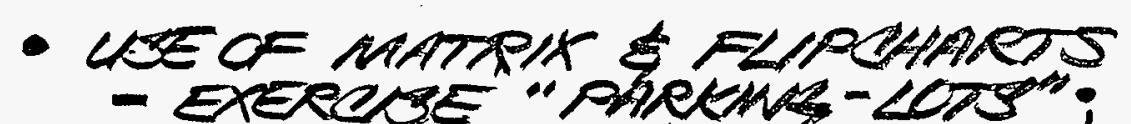

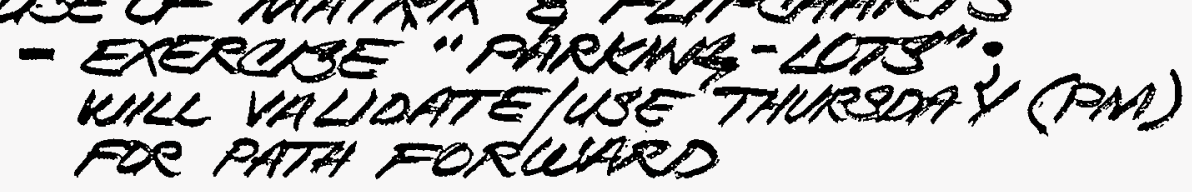

- KEXSTO SUCCESS

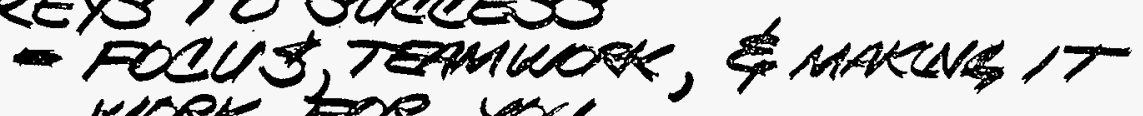
WOPK toe you.

HNIF 2576 RevO 